\title{
$R$-SETS AND CATEGORY
}

BY

RANA BARUA

\begin{abstract}
We prove some category theoretic results for $R$-sets much in the spirit of Vaught and Burgess. Since the proofs entail many results on $R$-sets and the $R$-operator, we have studied them in some detail and have formulated many results appropriate for our purpose in, perhaps, a more unified manner than is available in the literature. Our main theorem is the following: Any $R$-set in the product of two Polish spaces can be approximated, in category, uniformly over all sections, by sets generated by rectangles with one side an $R$-set and the other a Borel set. In fact, we prove a levelwise version of this result. For $C$-sets, this has been proved by $\mathrm{V}$. V. Srivatsa.
\end{abstract}

1. Introduction and preliminaries. The theory of $R$-sets and the $R$-operator, introduced by Kolmogorov almost half a century ago, has been studied extensively by Russian mathematicians $[10,12,13]$ and most of the basic properties have been deduced by them. However, it is only very recently that interest in the theory has been revived due mainly to the work of Hinman [7, 9] who developed the effective counterpart and showed that the effective hierarchies have deep interconnections with recursion-theoretic hierarchies. The introduction of Borel-programmable (BP) sets by Blackwell added a new dimension to the theory, and since then it has been shown by Burgess and Lockhart that the hierarchy obtained from BP-sets by iteration gives precisely the $R$-sets [6]. That two seemingly different definitions yield the same class of sets suggests that the $R$-sets form a natural class of subsets of the reals. Burgess has also obtained a different characterization for $R$-sets. He has proved that the entire hierarchy of $R$-sets can be obtained by applying the game quantifier to the "difference hierarchy" (of $\Delta_{3}^{0}$ ) obtained from sequences of $G_{8}$-sets [3]. Hinman (and also independently Aczel and Vaught, for the first level) first observed that the theory of inductive definability and games can be effectively used to study the hierarchy of $R$-sets $[8,1,19]$. These are the major tools employed by Burgess to prove most of his results on $R$-sets $[3,4]$. In this paper, to obtain our main result, viz. the approximation theorem, we have taken recourse to these methods.

$R$-sets in $X \times Y$, the product of two Polish spaces, are in general complicated sets and cannot be related to any reasonable product $\sigma$-field. For instance, as observed by B. V. Rao [16], $C$-sets in $\mathbf{R} \times \mathbf{R}$ need not belong to $\mathscr{L}(\mathbf{R}) \otimes \mathscr{L}(\mathbf{R})$, the product of the $\sigma$-fields of Lebesgue measurable sets. However, as shown by V. V. Srivatsa [17],

Received by the editors March 9, 1983.

1980 Mathematics Subject Classification. Primary 04A15; Secondary 03E15, 28A05, 03D55, 54H05.

$K e y$ words and phrases. Positive analytical operations, $\delta-s$ operation, inductive definability, game, $R$-operator, $R$-set. 
$C$-sets in $X \times Y$ can be "approximated" sectionwise, in the sense of measure and category, by sets in product $\sigma$-fields. His methods do not seem to generalize to $R$-sets. In this paper, we have shown that $R$-sets can be similarly approximated, in category, by sets in product $\sigma$-fields. This incidentally gives the selection theorem of Burgess (cf. [3]).

To obtain the approximation theorem, it is necessary to reprove some of Burgess' results in a more rigorous, accessible and, perhaps, transparent style. Our paper, therefore, serves a two-fold purpose.

The paper is organized as follows. Positive analytical operations and $\delta-s$ operations are introduced in $\$ 2$, and some of their elementary properties are discussed there. The papers of Kantorovitch and Livenson [10] give a detailed exposition of these operations. In $\S 3$, we have studied the operator $R$ of Kolmogorov and the more general operator $\mathscr{R}_{\mathscr{N}}$. In this section, we have also shown how inductive definability and games arise in the context of these operations. The theory of $R$-sets, studied in great detail by Lyapunov [12, 13], is treated in $\S 4$. In this section, we have given a proof of the pre-well-ordering property enjoyed by the classes $c \mathscr{R}^{\rho}\left(\rho<\omega_{1}\right)$ via the comparison of indices lemma. The proof of this lemma is much along the lines of the Kunen-Aczel theorem (cf. [14]). The comparison of indices lemma is crucial for our purpose since it helps in computing the complexity of the winning strategy for the existential player in the game associated with the operator $R$. This is done in $\$ 5$. Here we have also obtained a decomposition of $E^{*}=\left\{x: E^{x}\right.$ is comeager $\}$ for sets $E \in \Sigma_{1}^{\Phi^{*}}$, analogous to the one obtained by Vaught for analytic sets [19] and for $\mathscr{R}^{1}$-sets obtained by Burgess and Miller [5]. This immediately gives us the transfer theorem (cf. 5.4), which essentially computes $E^{*}$ when $E$ is computed by $R \Phi_{N}$, whenever the computation for $F^{*}$ for sets $F$ computed by $\Phi_{N}$ is known. $\S 6$ deals with a few applications of the transfer theorem, viz. the computation of $E^{*}$ for $R$-sets $E$. It is worth mentioning here that although our methods for computing $E^{*}$ are implicit in Burgess' proof for the same (cf. [5]), he computes $E^{*}$ only for $\mathscr{R}^{1}$-sets $E$, as any computation for higher levels using his methods will involve great notational complexities. By restricting ourselves to certain games of length $\omega$ and isolating the "core" of his proof (viz. the transfer theorem) we have been able to compute $E^{*}$ for all levels of the hierarchy of $R$-sets by a simple inductive argument. These computations yield sets in product $\sigma$-fields which "approximate" sectionwise (in the sense of category) $R$-sets in two dimensions. This is done in $\$ 7$. Incidentally, in this section, we have proved a slightly stronger version of the Game Formula of Kechris (cf. [11]) needed for our purpose.

For our notation and terminology we shall mainly follow Moschovakis [15]. The letter $\omega$ will denote the set of natural numbers and $\omega^{\omega}$ the set of all sequences of natural numbers equipped with the product of discrete topologies. Letters $\alpha, \beta, \gamma$, $\delta, \ldots$ will serve as variables over $\omega^{\omega}$ and $\eta, \xi, \ldots$ as variables over $2^{\omega}$. Seq will denote the set of sequence numbers of all finite sequences of natural numbers. We will mainly use $s, t, u, v$ to denote sequence numbers. We fix a base $\Sigma(s)$ for the topology of $\omega^{\omega}$, where

$$
\Sigma(s)=\left\{\alpha \in \omega^{\omega}: \bar{\alpha}(\ln (s))=s\right\} .
$$


If $s$ and $t$ are sequence numbers, we write $s \prec t$ if $s=t \uparrow i$ for some $i<\ln (s)$; $s * t$ or $\dot{s} t$ is the catenation of $s$ and $t$. If $s=\left\langle a_{0}, \ldots, a_{k-1}\right\rangle$, then $(s)_{i}=a_{i}$ for $i<k$.e or \langle\rangle will denote the empty sequence as well as its code.

If $\mathscr{F}$ is a collection of subsets of $X$, then $\sigma(\mathscr{F})$ denotes the $\sigma$-field generated by $\mathscr{F}$. If $\mathscr{B}$ and $\mathscr{C}$ are $\sigma$-fields on $T$ and $X$, respectively, $\mathscr{B} \otimes \mathscr{C}$ denotes the product $\sigma$-field. For $X$ a separable metric, $\mathscr{B}_{X}$ denotes its Borel $\sigma$-field.

Given a monotone set relation $\Gamma(w, x, A)$, where $w$ varies over $W$ and $A$ varies over subsets of $W, \Gamma_{x}$ denotes the induced set operation

$$
\Gamma_{x}(A)=\{w \in W: \Gamma(w, x, A)\} ; \quad x \in X .
$$

$\Gamma_{x}^{\mu}$ denotes the $\mu$ th iterate, viz.

$$
\Gamma_{x}^{\mu}=\Gamma_{x}\left(\bigcup_{\nu<\mu} \Gamma_{x}^{\nu}\right)
$$

We define

$$
\Gamma_{x}^{\infty}=\bigcup_{\mu} \Gamma_{x}^{\mu}
$$

and put

$$
w \in \Gamma_{x}^{<\mu} \leftrightarrow(\exists \nu<\mu)\left[w \in \Gamma_{x}^{\nu}\right]
$$

The fixed point of $\Gamma$ is

$$
\Gamma^{\infty}=\left\{(w, x): w \in \Gamma_{x}^{\infty}\right\}
$$

and is called the relation built up by $\Gamma$. We shall use elementary facts on inductive definability and games as found in [15]. Note that all unexplained notation and terminology is from Moschovakis [15].

The author wishes to express his gratitude to Professor Ashok Maitra for introducing $R$-sets to him and for general guidance at every stage in the preparation of this paper. He is also grateful to V. V. Srivatsa for innumerable discussions. The influence of $J$. P. Burgess is evident on every page.

2. Positive analytical operations. In this section, we shall discuss positive analytical operations and some of their properties needed for our purpose.

Let $X$ be a nonempty set and $N \subseteq \omega^{\omega}$. Let $\left\{E_{n}: n \in \omega\right\}$ be a sequence of subsets of $X$.

DeFINITION 2.1. The $\delta-s$ operation with base $N$ is defined by

$$
\Phi_{N}\left(\left\{E_{n}: n \in \omega\right\}\right)=\bigcup_{\alpha \in N} \bigcap_{k=0}^{\infty} E_{\alpha(k)} .
$$

In most cases we shall take the base set $N \subseteq 2^{\omega}$ so that

$$
\Phi_{N}\left(\left\{E_{n}: n \in \omega\right\}\right)=\bigcup_{\eta \in N} \bigcap_{n \in \eta} E_{n} .
$$

To avoid trivialities, we shall always assume $\varnothing \notin N$ and $N \neq \varnothing$.

EXAMPLES. If $N=\left\{\lambda n \bar{\alpha}(n): \alpha \in \omega^{\omega}\right\}$, then $\Phi_{N}=$ operation $\mathscr{A}$. If

$$
N=\left\{\alpha \in \omega^{\omega} \mid \operatorname{Range}(\alpha) \text { is infinite }\right\},
$$

then $\Phi_{N}=\lim \sup$. If $N=\{(n, n, \ldots) \mid n \in \omega\}$, then $\Phi_{N}=U$ (countable). 
An operation over $X$ is a function $\Phi: \mathscr{P}(x)^{\omega} \rightarrow \mathscr{P}(x)$.

DEFINITION 2.2. An operation over $X$ is said to be a positive analytical operation if

(a) $\Phi$ is nonconstant and

(b) $x \in \Phi\left(\left\{E_{n}: n \in \omega\right\}\right) \& y \notin \Phi\left(\left\{F_{n}: n \in \omega\right\}\right) \rightarrow(\exists n)\left(x \in E_{n} \& y \notin F_{n}\right)$.

Clearly, $\cup, \mathscr{A}$, limsup are positive analytical operations. A positive analytical operation on constant sequences takes on the constant value and is isotone i.e. for any families $\left\{F_{n}: n \in \omega\right\}$ and $\left\{G_{n}: n \in \omega\right\}$, if $(\forall n)\left(F_{n} \subseteq G_{n}\right)$, then $\Phi\left(\left\{F_{n}: n \in \omega\right\}\right)$ $\subseteq \Phi\left(\left\{G_{n}: n \in \omega\right\}\right)$.

Given a positive analytical operation $\Phi$ over $X$, one can define a positive analytical operation $\Phi^{\prime}$ over any set $Y$ as follows. For any family $\left\{F_{n}: n \in \omega\right\}$ of subsets of $Y$ and $y \in Y$, put

$$
\begin{aligned}
y \in \Phi^{\prime}\left(\left\{F_{n}: n \in \omega\right\}\right) \leftrightarrow & \text { there is a family }\left\{E_{n}: n \in \omega\right\} \text { of subsets of } X \\
& \text { and } x \in X \text { such that } x \in \Phi\left(\left\{E_{n}: n \in \omega\right\}\right) \\
& \text { and }(\forall n)\left(x \in E_{n} \rightarrow y \in F_{n}\right) .
\end{aligned}
$$

It is easy to check that $\Phi^{\prime}$ is positive analytical and $\Phi^{\prime}=\Phi$ when $Y=X$. The operation $\Phi^{\prime}$ is called the extension of $\Phi$ over $Y$. Henceforth we shall use the same symbol $\Phi$ to denote a positive analytical operation together with all its extensions.

Notice that a $\delta-s$ operation is a positive analytical operation (over any set $X$ ). The converse is true and follows from the following.

Proposition. Let $\Phi$ be a positive analytical operation over $X$. Then $\Phi=\Phi_{N}$ for some $N \subseteq 2^{\omega}\left(N \subseteq \omega^{\omega}\right)$.

Proof. Let

$$
\begin{aligned}
D_{i} & =\left\{\eta \in 2^{\omega} \mid i \in \eta\right\}, \quad \text { or, } \\
& =\left\{\alpha \in \omega^{\omega} \mid i \in \operatorname{Range}(\alpha)\right\}, \quad i \in \omega .
\end{aligned}
$$

Put $N=\Phi\left(\left\{D_{i}: i \in \omega\right\}\right)$. It is easy to check that $\Phi=\Phi_{N}$. The set $N$ obtained above is called the canonical base for $\Phi$.

Definition 2.3. A base $N \subseteq \omega^{\omega}$ is complete if $N=\Phi_{N}\left(\left\{D_{n}: n \in \omega\right\}\right)$, when $D_{n}$ is defined as in the Proposition. Thus the canonical base for a positive analytical operation is complete.

Equivalently, a base $N \subseteq 2^{\omega}$ is complete if

$$
\eta \in N \& \eta \subseteq \eta^{\prime} \subseteq \omega \rightarrow \eta^{\prime} \in N .
$$

If $\tilde{N}=\Phi_{N}\left(\left\{D_{n}: n \in \omega\right\}\right)$, then $\tilde{N}$ is complete (called the completion of $N$ ) and, moreover, $\Phi_{\tilde{N}}=\Phi_{N}$.

Definition 2.4. For any operation $\Phi$, the dual $\Phi^{0}$ is defined by

$$
\Phi^{0}\left(\left\{E_{n}: n \in \omega\right\}\right)=\left[\Phi_{N}\left(\left\{E_{n}^{c}: n \in \omega\right\}\right)\right]^{c}
$$

e.g. $\cup^{0}=\cap,(\lim \sup )^{0}=\lim \inf , \mathscr{A}^{0}=\Gamma$, where

$$
\Gamma\left(\left\{E_{n}: n \in \omega\right\}\right)=\left\{x:(\forall \alpha)(\exists n)\left(x \in E_{\bar{\alpha}(n)}\right)\right\} .
$$


If $\Phi_{N}$ is a $\delta-s$ operation with base $N \subseteq 2^{\omega}$, then the canonical base $N^{0}$ of its dual is given by

$$
\begin{aligned}
N^{0} & =\left\{\eta \in 2^{\omega}: \eta \cap \eta^{\prime} \neq \varnothing \text { for every } \eta^{\prime} \in N\right\} \\
& =\left\{\eta \in 2^{\omega}: \eta^{c} \notin N\right\}, \quad \text { if } N \text { is complete. }
\end{aligned}
$$

Plainly, $N^{0}$ is always complete. Thus, for any family $\left\{E_{n}: n \in \omega\right\}$,

$$
\left(\exists \eta \in N^{0}\right)(\forall n \in \eta)\left[x \in E_{n}\right] \leftrightarrow(\forall \eta \in N)(\exists n \in \eta)\left[x \in E_{n}\right] .
$$

If $N$ is complete, $N^{00}=N$ and hence

$$
\left(\forall \eta \in N^{0}\right)(\exists n \in \eta)\left[x \in E_{n}\right] \leftrightarrow(\exists \eta \in N)(\forall n \in \eta)\left[x \in E_{n}\right] .
$$

3. The $R$-operator. Although the $R$-operator was first introduced by Kolmogorov, the first published account of the theory appeared in [10] and further results obtained in [12, 13]. Lyapunov also studied the hierarchies of $R$-sets (cf. \$4) and obtained most of their properties. The interconnection between $R$-operators and games was first noticed by Hinman [8] (and also independently by Aczel [1]). Hinman also developed the effective theory and did most of the groundwork. Much of the material in this section is adapted from these sources.

Definition 3.1. Let $\mathscr{N}=\left\{N_{p} \subseteq 2^{\omega}: p \in \omega\right\}$ be a sequence of nonempty bases. $\theta \subseteq \omega$ is called an $\mathscr{N}$-chain if

(a) $e \in \theta$,

(b) $s \in \theta$ and $t \prec s \rightarrow t \in \theta$,

(c) $s \in \theta \rightarrow\{n: \hat{s}\langle n\rangle \in \theta\} \in N_{s}$.

Put $\Theta_{\mathscr{N}}=\{\theta: \theta$ is an $\mathscr{N}$-chain $\}$.

$\mathscr{R}_{\mathscr{N}}$ is the set operation defined by

$$
\mathscr{R}_{\mathcal{N}}\left(\left\{E_{n}: n \in \omega\right\}\right)=\bigcup_{\theta \in \Theta_{\mathcal{N}}} \bigcap_{s \in \theta} E_{s}
$$

Clearly, $\mathscr{R}_{\mathscr{N}}$ is a $\delta-s$ operation with base $\Theta_{\mathscr{N}}$.

If $N_{p}=N$ for each $p$, then $\mathscr{R}_{\mathscr{N}}$ is denoted by $R \Phi_{N}$ and its base by $R N$. An $\mathcal{N}$-chain will then be called an $N$-chain.

EXAMPLES. Let $N=\{\{n\}: n \in \omega\}$ so that $\Phi_{N}=U$ and put $\mathscr{N}=\{N\}$. Clearly, an $\mathscr{N}$-chain is any set of the form $\{\bar{\alpha}(n): n \in \omega\}$. Thus $R \cup=R \Phi_{N}=\mathscr{A}$. If $N=\{\omega\}$, then $\Phi_{N}=\cap$ (countable) and the only $\mathscr{N}$-chain is $\omega$, so $R \cap=\cap$.

Definition 3.2. Let $\Phi_{N}$ and $\Phi_{M}$ be two $\delta-s$ operations with bases $N, M \subseteq 2^{\omega}$. The composed operation $\Psi$ is given by

$$
\Psi\left(\left\{F_{n}: n \in \omega\right\}\right)=\Phi_{N}\left(\left\{\Phi_{M}\left(\left\{F_{\langle p, n\rangle}: n \in \omega\right\}\right): p \in \omega\right\}\right) .
$$

$\Psi$ is sometimes denoted by $\Phi_{N} \Phi_{M}$. By the characterization lemma, $\Psi$ is a $\delta-s$ operation whose canonical base we shall denote by $N M$. Thus $\Psi=\Phi_{N} \Phi_{M}=\Phi_{N M}$ and

$$
\eta \in N M \leftrightarrow\left(\exists \eta_{1} \in N\right)\left(\forall n_{1} \in \eta_{1}\right)\left(\exists \xi_{1} \in M\right)\left(\forall m_{1} \in \xi_{1}\right)\left[\left\langle n_{1}, m_{1}\right\rangle \in \eta\right] .
$$

Henceforth, for simplicity, we shall take $X=\omega^{\omega}$ or $\left(\omega^{\omega}\right)^{k} \times \omega^{1}$, although most of the results hold for a general Polish space. 
Definition 3.3. For any operation $\Phi$, let $\Sigma_{1}^{\Phi}$ be the class of relations of the form $\Phi\left(\left\{F_{n}: n \in \omega\right\}\right)$ with all $F_{n}$ clopen, $\Pi_{1}^{\Phi}$ the class of complements of such relations, and $\Delta_{1}^{\Phi}=\Sigma_{1}^{\Phi} \cap \Pi_{1}^{\Phi}$. Then $\Sigma_{1}^{\cup}=\Sigma_{1}^{0}$ and $\Sigma_{1}^{\infty}=\Sigma_{1}^{1}$.

If $\Phi=\Phi_{N}$, then define $\Phi^{*}=R \Phi_{N N^{0}}$.

The next two lemmas show a close connection between $R$-operators, inductive definability and games.

LemMA 3.4. (a) Suppose $F=R \Phi_{N}\left(\left\{F_{n}: n \in \omega\right\}\right)$. Then

$$
\begin{array}{r}
x \in F \leftrightarrow\left(\exists \eta_{0} \in N\right)\left(\forall n_{0} \in \eta_{0}\right)\left(\exists \eta_{1} \in N\right)\left(\forall n_{1} \in \eta_{1}\right) \cdots \\
\cdots(\forall k)\left[x \in F_{\left\langle n_{0}, n_{1} \ldots n_{k-1}\right\rangle}\right] .
\end{array}
$$

(b) If $E=\Phi_{N}^{*}\left(\left\{E_{n}: n \in \omega\right\}\right)$, then

$$
\begin{aligned}
x \in E \leftrightarrow & \left(\exists \eta_{0} \in N\right)\left(\forall n_{0} \in \eta_{0}\right)\left(\forall \xi_{0} \in N\right)\left(\exists m_{0} \in \xi_{0}\right)\left(\exists \eta_{1} \in N\right)\left(\forall n_{1} \in \eta_{1}\right) \\
& \left(\forall \xi_{1} \in N\right)\left(\exists m_{1} \in \xi_{1}\right) \cdots \cdots(\forall k)\left[x \in E_{\left\langle\left\langle n_{0}, m_{0}\right\rangle \ldots\left\langle n_{h-1}, m_{k-1}\right\rangle\right\rangle}\right] .
\end{aligned}
$$

(The right-hand side of each equivalence is interpreted in terms of games between two players $\forall$ and $\exists$.)

Proof. Clearly (b) follows from (a) and the fact that

$$
\eta \in N N^{0} \leftrightarrow\left(\exists \eta_{1} \in N\right)\left(\forall n_{1} \in \eta_{1}\right)\left(\forall \xi_{1} \in N\right)\left(\exists m_{1} \in \xi_{1}\right)\left[\left\langle n_{1}, m_{1}\right\rangle \in \eta\right] .
$$

To prove the first assertion, fix $x$ and suppose $x \in F$. Get an $N$-chain $\theta \in R N$ such that $(\forall s \in \theta)\left[x \in F_{s}\right]$. Now, consider the following strategy for $\exists$. As his first move $\exists$ plays $\eta_{0}=\{n:\langle n\rangle \in \theta\}$ which is clearly in $N$. Any response $n_{0} \in \eta_{0}$ by $\forall$ gives a set $\eta_{1}=\left\{n:\left\langle n_{0}, n\right\rangle \in \theta\right\} \in N$ and $\exists$ should play $\eta_{1}$ as his next move. If $\forall$ then plays $n_{1} \in \eta_{1}$, we still have $\left\langle n_{0}, n_{1}\right\rangle \in \theta$ and $\exists$ responds with $\eta_{2}=\left\{n:\left\langle n_{0}, n_{1}, n\right\rangle\right.$ $\in \theta\}$. If $\exists$ follows this strategy, then clearly for any $k,\left\langle n_{0}, n_{1}, \ldots, n_{k-1}\right\rangle \in \theta$ and so $x \in F_{\left\langle n_{0} \ldots, n_{h-1}\right\rangle}$. Hence it is a winning strategy for $\exists$ in the game (*).

For the converse implication suppose $\sigma$ is a winning strategy for $\exists$. Let $\theta$ be the set of sequences $\left\langle n_{0}, \ldots, n_{k-1}\right\rangle$ of first $k$ possible moves of player $\forall$ when $\exists$ follows this strategy $\sigma$. Clearly $\theta$ is an $N$-chain and since $\sigma$ is a winning strategy for $\exists$,

$$
(\forall k)\left[\left\langle n_{0}, \ldots, n_{k-1}\right\rangle \in \theta \rightarrow x \in F_{\left\langle n_{0}, n_{1} \ldots, n_{k-1}\right\rangle}\right] .
$$

Hence $x \in R \Phi_{N}\left(\left\{F_{n}: n \in \omega\right\}\right)=F$.

REMARK. It follows from Lemma 3.4 that our definition of $\Phi^{*}$ is equivalent (cf. Definition 3.9) to that introduced in [8, V. 4].

The next result is due to Hinman [8].

THEOREM 3.5. For any positive analytical operation $\Phi$ and any $E \subseteq\left(\omega^{\omega}\right)^{k} \times \omega^{1}$ in $\Pi_{1}^{\Phi^{*}}$, there exists a (monotone) inductive operator $\Gamma$ such that for all $x$

$$
E(x) \leftrightarrow e \in \Gamma_{x}^{\infty} .
$$

Proof. Let $\left\{E_{s}: s \in \omega\right\}$ be a family of clopen subsets of $\left(\omega^{\omega}\right)^{k} \times \omega^{1}$ such that $x \notin E \leftrightarrow x \in \Phi^{*}\left(\left\{E_{s}: s \in \omega\right\}\right)$. 
Let $N \subseteq 2^{\omega}$ be the canonical base for $\Phi$. Then $\Phi^{*}=R \Phi_{N N^{0}}$. Define a set relation operative on $\omega$ as follows:

(4)

$s \in \Gamma_{x}(A) \leftrightarrow x \notin E_{s} \vee(\forall \eta \in N)(\exists n \in \eta)(\exists \xi \in N)(\forall m \in \xi)[\hat{s}\langle\langle n, m\rangle\rangle \in A]$.

Clearly, $\Gamma(s, x, A)$ is a monotone set relation. Put $E^{s}=\Phi^{*}\left(\left\{E_{s * t}: t \in \omega\right\}\right)$. One can easily see that $E^{e}=E^{c}$ and $E^{s} \subseteq E_{s}$. We claim that for all $s$,

$x \notin E^{s} \leftrightarrow s \in \Gamma_{x}^{\infty}$

and the result follows by putting $s=\langle\rangle$. We shall prove the implication $(\leftarrow)$ by induction. If $s \in \Gamma_{x}^{0}$, then $x \notin E_{s} \supseteq E^{s}$. Now suppose $s \in \Gamma_{x}^{\mu}, \mu>0$. Then

$x \notin E_{s} \vee(\forall \eta \in N)(\exists n \in \eta)(\exists \xi \in N)(\forall m \in \xi)\left[s *\langle\langle n, m\rangle\rangle \in \Gamma_{x}^{<\mu}\right]$.

If $x \notin E_{s}$, we are done; otherwise by induction hypothesis

$$
(\forall \eta \in N)(\exists n \in \eta)(\exists \xi \in N)(\forall m \in \xi)\left[x \notin E^{s *\langle\langle n, m\rangle\rangle}\right]
$$

which by Lemma 3.4 (and determinacy) implies

$$
\begin{aligned}
& (\forall \eta \in N)(\exists n \in \eta)(\exists \xi \in N)(\forall m \in \xi)\left\{\left(\forall \eta_{1} \in N\right)\left(\exists n_{1} \in \eta_{1}\right)\right.
\end{aligned}
$$

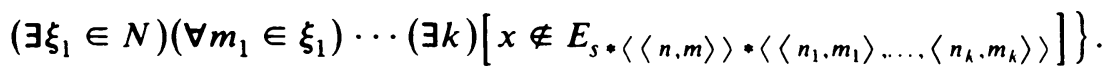

This clearly implies

$$
\begin{gathered}
\left(\forall \eta_{0} \in N\right)\left(\exists n_{0} \in \eta_{0}\right)\left(\exists \xi_{0} \in N\right)\left(\forall m_{0} \in \xi_{0}\right)\left(\forall \eta_{1} \in N\right)\left(\exists n_{1} \in \eta_{1}\right) \\
\left(\exists \xi_{1} \in N\right)\left(\forall m_{1} \in \xi_{1}\right) \cdots(\exists k)\left[x \notin E_{\left.s *\left\langle\left\langle n_{0}, m_{0}\right\rangle \ldots,\left\langle n_{k-1}, m_{k-1}\right\rangle\right\rangle\right]}\right.
\end{gathered}
$$

and thus $x \notin R \Phi_{N N^{0}}\left(\left\{E_{s * t} ; t \in \omega\right\}\right)=E^{s}$, by Lemma 3.4 again.

Conversely, let $s \notin \Gamma_{x}^{\infty}$. We shall show that $x \in E^{s}$ i.e.,

$(* *) \quad\left(\exists \eta_{0} \in N\right)\left(\forall n_{0} \in \eta_{0}\right)\left(\forall \xi_{0} \in N\right)\left(\exists m_{0} \in \xi_{0}\right)\left(\exists \eta_{1} \in N\right)\left(\forall n_{1} \in \eta_{1}\right)$

$$
\left(\forall \xi_{1} \in N\right)\left(\exists m_{1} \in \xi_{1}\right) \cdots(\forall k)\left[x \in E_{\left.s *\left\langle\left\langle n_{0}, m_{0}\right\rangle \ldots,\left\langle n_{k-1}, m_{k-1}\right\rangle\right\rangle\right] .}\right.
$$

Since $s \notin \Gamma_{x}\left(\Gamma_{x}^{\infty}\right)$, by definition of $\Gamma, x \in E_{s}$ and moreover,

$$
\left(\exists \eta_{0} \in N\right)\left(\forall n_{0} \in \eta_{0}\right)\left(\forall \xi_{0} \in N\right)\left(\exists m_{0} \in \xi_{0}\right)\left[s *\left\langle\left\langle n_{0}, m_{0}\right\rangle\right\rangle \notin \Gamma_{x}^{\infty}\right] .
$$

Now, $\exists$ can win the game (**) by adopting the following strategy. He picks $\eta_{0} \in N$ such that for any choice of $n_{0} \in \eta_{0}$ and $\xi_{0} \in N$, there is an $m_{0} \in \xi_{0}$ such that $s *\left\langle\left\langle n_{0}, m_{0}\right\rangle\right\rangle \notin \Gamma_{x}^{\infty}=\Gamma_{x}\left(\Gamma_{x}^{\infty}\right)$. Thus $x \in E_{s *\left\langle\left\langle n_{0}, m_{0}\right\rangle\right\rangle}$ and

$$
\left(\exists \eta_{1} \in N\right)\left(\forall n_{1} \in \eta_{1}\right)\left(\forall \xi_{1} \in N\right)\left(\exists m_{1} \in \xi_{1}\right)\left[s *\left\langle\left\langle n_{0}, m_{0}\right\rangle,\left\langle n_{1}, m_{1}\right\rangle\right\rangle \notin \Gamma_{x}^{\infty}\right] .
$$

$\exists$ then picks $\eta_{1} \in N$ such that for any choice of $n_{1} \in \eta_{1}$ and $\xi_{1} \in N$ made by $\forall$, there is an $m_{1} \in \xi_{1}$ (and $\exists$ plays such an $m_{1}$ ) such that $s *\left\langle\left\langle n_{0}, m_{0}\right\rangle,\left\langle n_{1}, m_{1}\right\rangle\right\rangle \notin$ $\Gamma_{x}^{\infty}$. Proceeding this way, $\exists$ has a strategy which ensures that for all $k, x \in$ $E_{s *\left\langle\left\langle n_{0}, m_{0}\right\rangle \ldots,\left\langle n_{k-1}, m_{k-1}\right\rangle\right\rangle}$ and so $\exists$ wins the game $(* *)$. Consequently, $x \in E^{s}$.

REMARK. If $E$ is such that $x \notin E \leftrightarrow x \in R \Phi_{N}\left(\left\{E_{s}: s \in \omega\right\}\right)$, then the inductive operator takes a simpler form, viz.,

$$
s \in \Gamma_{x}(A) \leftrightarrow x \notin E_{s} \vee(\forall \eta \in N)(\exists n \in \eta)[s *\langle n\rangle \in A]
$$


More generally, if $\mathscr{N}=\left\{N_{p}: p \in \omega\right\}$ is a sequence of bases and

$$
E^{c}=\mathscr{R}_{\mathscr{N}}\left(\left\{E_{s}: s \in \omega\right\}\right),
$$

then we take the following inductive operator

$$
s \in \Gamma_{x}(A) \leftrightarrow x \notin E_{s} \vee\left(\forall \eta \in N_{s}\right)(\exists n \in \eta)[s *\langle n\rangle \in A],
$$

and the conclusion of the above theorem still holds.

The inductive operator (4) (or (6) or (7)) is called the canonical inductive operator associated with $\left\{E_{s}: s \in \omega\right\}$ and $N$ (or $\mathscr{N}$ ).

The above characterization theorem yields a decomposition of sets in $\Pi_{1}^{\Phi^{*}}\left(\Sigma_{1}^{\Phi^{*}}\right)$ into simpler sets as is evident from the next theorem.

Definition 3.6. For any operation $\Phi, \nabla(\Phi)$ is the smallest class of relations containing clopen relations and closed under $\Phi$ and $\Phi^{0}$.

Thus $\nabla(\cup)=\nabla(\cap)=\Delta_{1}^{1}$.

Let $E \in \Sigma_{1}^{\Phi^{*}}$ and suppose $E=\Phi^{*}\left(\left\{E_{s}: s \in \omega\right\}\right)$. Let $N$ and $\Gamma$ be as above. Then by $3.5, E(x) \leftrightarrow e \notin \Gamma_{x}^{\infty}$. Set

$$
E_{s}^{\mu}=\left\{x: s \notin \Gamma_{x}^{\mu}\right\}
$$

Then $E=\bigcap_{\mu<\omega_{1}} E_{e}^{\mu}$. Now define

$$
T^{\mu}=\bigcup_{s \in \omega}\left(E_{s}^{\mu}-E_{s}^{\mu+1}\right)
$$

It is easy to prove by induction on $\mu$ that for all $\mu<\omega_{1}, s \in \omega, E_{s}^{\mu}$ and $T^{\mu}$ are in $\nabla(\Phi)$. Then we have

THEOREM 3.7. $E=\cup_{\mu<\omega_{1}}\left(E_{e}^{\mu}-T^{\mu}\right)=\bigcap_{\mu<\omega_{1}} E_{e}^{\mu}$.

Proof. Let $x \in E$. Define

$$
\beta(s)=\left\{\begin{array}{l}
\text { least ordinal } \rho \text { such that } x \notin E_{s}^{\rho} \quad \text { if }(\exists \rho)\left(x \notin E_{s}^{\rho}\right), \\
0 \text { otherwise. }
\end{array}\right.
$$

Let $\omega_{1}>\rho_{0}>\beta(s), \forall s$. Then $(\forall s)\left[x \in E_{s}^{\rho_{0}} \leftrightarrow x \in E_{s}^{\rho_{0}+1}\right]$. Consequently, $x \notin T^{\rho_{0}}$ and thus $x \in E_{e}^{\rho_{0}}-T^{\rho_{0}}$.

Conversely, suppose for some $\rho_{0}<\omega_{1}, x \in E_{e}^{\rho_{0}}-T^{\rho_{0}}$. Then

$$
(\forall s)\left[x \in E_{s}^{\rho_{0}} \leftrightarrow x \in E_{s}^{\rho_{0}+1}\right] .
$$

One can check by induction that

$$
\left(\forall \rho>\rho_{0}\right)(\forall s)\left[x \in E_{s}^{\rho_{0}} \leftrightarrow x \in E_{s}^{\rho}\right] .
$$

So in particular, $x \in \cap_{\rho>\rho_{0}} E_{e}^{\rho}=\bigcap_{\rho<\omega_{1}} E_{e}^{\rho}=E$.

Standard arguments using the above decomposition and the countable chain condition yield the following (cf. [8]).

THEOREM 3.8. If $\Phi$ preserves measurability ( preserves the Baire property), then so does $\Phi^{*}$.

Definition 3.9. For any two operations $\Phi$ and $\Psi, \Phi$ subsumes $\Psi(\Phi \geqslant \Psi)$ if there is a function $f: \omega \rightarrow \omega$ such that for any family $\left\{F_{n}: n \in \omega\right\}$,

$$
\Psi\left(\left\{F_{n}: n \in \omega\right\}\right)=\Phi\left(\left\{F_{f(n)}: n \in \omega\right\}\right) .
$$


$\Phi$ and $\Psi$ are said to be equivalent $(\Phi \sim \Psi)$ if $\Phi \geqslant \Psi$ and $\Psi \geqslant \Phi$. For example, $\mathscr{A}$ subsumes both $U$ and $\cap$.

DEFINITION 3.10. A positive analytical operation $\Phi$ is said to be normal if there is a function $g$ such that for any family $\left\{F_{n}: n \in \omega\right\}$,

$$
\Phi\left(\left\{\Phi\left(\left\{F_{\langle p, q\rangle}: q \in \omega\right\}\right): p \in \omega\right\}\right)=\Phi\left(\left\{F_{g(n)}: n \in \omega\right\}\right) .
$$

We shall omit the proof of the next lemma which can be found in $[7,9]$.

LEMMA 3.11. For any operations $\Phi$ and $\Psi$
(a) $\Phi^{00}=\Phi$;
(b) $\Phi \geqslant \Psi \rightarrow \Phi^{0} \geqslant \Psi^{0}$;
(c) $\Phi \circ \Phi^{0} \geqslant \Phi, \Phi^{0}$;
(d) $R \Phi \geqslant \Phi$;
(e) $\Phi \geqslant \Psi \rightarrow R \Phi \geqslant R \Psi$;
(f) $R \Phi \geqslant \Psi$ and $R \Phi \geqslant \Psi^{0} \rightarrow R \Phi \geqslant \Psi \cdot \Psi^{n}$;
(g) $R \Phi \sim R R \Phi$;
(h) $R \Phi$ is normal.

4. The $R$-sets. We shall first construct a sequence $\left\{R_{\rho}: p<\omega_{1}\right\}$ of positive analytical operations by induction as follows. Put $R_{0}=\mathscr{A}$ and having defined $R_{\rho}$, put

$$
R_{\rho+1}=R_{\rho}^{*} \text {. }
$$

If $\lambda$ is the limit, choose a sequence $\rho_{i} \uparrow \lambda$ and set, for any family $\left\{E_{n}: n \in \omega\right\}$,

$$
\Phi\left(\left\{E_{n}: n \in \omega\right\}\right)=\bigcap_{i=0}^{\infty} \Phi_{N_{p_{i}} N_{p_{1}}^{0}}\left(\left\{E_{\langle i, m\rangle}: m \in \omega\right\}\right),
$$

where $N_{\rho_{1}}$ is the canonical base for $R_{\rho_{l}}$. Then define

$$
R_{\lambda}=\Phi^{*} \text {. }
$$

Note that any other sequence $\rho_{i}^{\prime} \uparrow \lambda$ gives rise to an equivalent operation by Lemma 3.11. Also, it is easy to check that $R_{\rho} \geqslant R_{\rho^{\prime}}$ if $\rho \geqslant \rho^{\prime}$.

For each $\rho<\omega_{1}$, let $\mathscr{R}^{\rho}=\Sigma_{1}^{R_{\rho}}$ and $B \mathscr{R}^{\rho}=\Delta_{1}^{R^{\rho}}$. Let $\mathscr{B} \mathscr{R}^{\rho}$ be the least class containing clopen relations and closed under $R_{\rho}$ and complementation. Thus, for instance, $\mathscr{R}^{0}=\Sigma_{1}^{1}, B \mathscr{R}^{0}=\Delta_{1}^{1}$ and $\mathscr{B} \mathscr{R}^{0}=C$-sets of Selivanovskii. Finally, set

$$
\mathscr{R}=\bigcup_{\rho<\omega_{1}} \mathscr{R}^{\rho} .
$$

Members of $\mathscr{R}$ are known classically as the $R$-sets. It is not difficult to see that for each $\rho$,

$$
\mathscr{R}^{\rho} \subseteq \mathscr{B} \mathscr{R}^{\rho} \subseteq B \mathscr{R}^{\rho+1} \subseteq \mathscr{R}^{\rho+1} .
$$

In fact, the inclusions can be shown to be strict (cf. $[12,13])$.

The following is immediate.

LEMMA 4.1. For every $\rho, R_{\rho}$ is normal and $R R_{\rho} \sim R_{\rho}$. 
For each $\rho$, the class $\mathscr{B} \mathscr{R}^{\rho}$ can be decomposed into a hierarchy just as in the case of the Borel class. Suppose $R^{\rho}=\Phi^{*}$. We set $\mathscr{R}_{0}^{\rho}=\mathscr{R}^{\rho}$ and take

$$
\mathscr{R}_{\mu}^{\rho}=R^{\rho}\left[\left(\bigcup_{\nu<\mu} \mathscr{R}_{\nu}^{\rho}\right)^{c}\right], \quad B \mathscr{R}_{\mu}^{\rho}=\left\{E: E, E^{c} \in \mathscr{R}_{\mu}^{\rho}\right\} .
$$

$\mathscr{B} \mathscr{R}_{\mu}^{\rho}$ is the smallest class containing $\mathscr{R}_{\mu}^{\rho}$ and closed under $\Phi$ and complementation. It is immediate that these classes are included in $\mathscr{B} \mathscr{R}^{\rho}$ and indeed that $\mathscr{B} \mathscr{R}^{\rho}=$ $\bigcup_{\mu<\omega_{1}} \mathscr{R}_{\mu}^{\rho}$. As above we have, for each $\rho, \mu<\omega_{1}$,

$$
\mathscr{R}_{\mu}^{\rho} \subsetneq \mathscr{B} \mathscr{R}_{\mu}^{\rho} \subsetneq B \mathscr{R}_{\mu+1}^{\rho} \subsetneq \mathscr{R}_{\mu+1}^{\rho} .
$$

We shall now show that each class $c \mathscr{R}^{\rho}=\Pi_{1}^{R}$ has the pre-well-ordering property. The key to this is the following lemma.

If $E=\mathscr{R}_{\mathscr{N}}\left(\left\{E_{n}: n \in \omega\right\}\right)$, where $\mathscr{N}=\left\{N_{p}: p \in \omega\right\}$ and $\Gamma$ the canonical inductive operator asociated with $\left\{E_{n}\right\}$ and $\mathscr{N}$, then we have $x \notin E \leftrightarrow e \in \Gamma_{x}^{\infty}$. Put

$$
|(s, x)|_{\Gamma}= \begin{cases}\text { least } \rho \text { such that } s \in \Gamma_{x}^{\rho} & \text { if such } \rho \text { exists, } \\ \omega_{1} & \text { otherwise. }\end{cases}
$$

Thus $|(e, x)|_{\Gamma}<\omega_{1} \leftrightarrow x \notin E$.

LEMMA 4.2 (COMPARISON OF INDICES). Let $\mathscr{E}=\left\{E_{n}: n \in \omega\right\}$ and $\mathscr{F}=\left\{F_{n}: n \in \omega\right\}$ be two families of subsets of $X$ and further assume that $\mathscr{F}$ is regular, i.e., $F_{t} \subseteq F_{s}$ if $s \prec t$. Let $\mathscr{N}=\left\{N_{p}: p \in \omega\right\}, \mathscr{M}=\left\{M_{p}: p \in \omega\right\}$ be two sequences of bases. Define a sequence of bases $\left\{K_{s}: s \in \omega\right\}$ as follows. If $s=\left\langle\left\langle n_{0}, m_{0}\right\rangle, \ldots,\left\langle n_{k}, m_{k}\right\rangle\right\rangle$, then $K_{s}$ is the canonical base for the positive analytical operation $\Phi$ defined by

$$
\Phi\left(\left\{G_{n}: n \in \omega\right\}\right)=\Phi_{M_{\left\langle m_{0} \ldots \ldots m_{k}\right\rangle}^{0}}\left(\left\{\Phi_{N_{\left\langle n_{0} \ldots n_{k}\right\rangle}}\left(\left\{G_{\langle n, m\rangle}: n \in \omega\right\}\right): m \in \omega\right\}\right) .
$$

Otherwise, $K_{s}=\{\omega\}$. Let

$$
\begin{gathered}
\mathscr{K}=\left\{K_{s}: s \in \omega\right\}, \\
H_{s}=\left\{\begin{array}{l}
E_{\left\langle n_{0} \ldots \ldots n_{h}\right\rangle} \cup F_{\left\langle m_{0} \ldots, m_{h}\right\rangle}^{c} \text { if } s=\left\langle\left\langle n_{0}, m_{0}\right\rangle, \ldots,\left\langle n_{k}, m_{k}\right\rangle\right\rangle, \\
X \text { otherwise. }
\end{array}\right.
\end{gathered}
$$

Suppose $\Gamma$ is the canonical inductive operator associated with $\mathscr{E}$ and $\mathscr{N} ; \Delta$ the inductive operator associated with $\mathscr{F}$ and $\mathscr{M}$. Then,

$$
\left\{x:|(e, x)|_{\Gamma}<|(e, x)|_{\Delta}\right\}^{c}=\mathscr{R}_{\mathscr{X}}\left(\left\{H_{s}: s \in \omega\right\}\right) .
$$

ProOF. First note that

$\eta \in K_{\left\langle\left\langle n_{0}, m_{0}\right\rangle \ldots,\left\langle n_{1-1}, m_{1-1}\right\rangle\right\rangle}$

$$
\leftrightarrow\left(\exists \eta^{\prime} \in M_{\left\langle m_{0}, \ldots, m_{l-1}\right\rangle}^{0}\right)\left(\forall m \in \eta^{\prime}\right)\left(\exists \eta^{\prime \prime} \in N_{\left\langle n_{0}, \ldots, n_{t-1}\right\rangle}\right)\left(\forall n \in \eta^{\prime \prime}\right)[\langle n, m\rangle \in \eta] .
$$

The operators $\Gamma$ and $\Delta$ are as follows.

$$
\begin{aligned}
& s \in \Gamma_{x}(A) \leftrightarrow x \notin E_{s} \vee\left(\forall \eta \in N_{s}\right)(\exists n \in \eta)[s *\langle n\rangle \in A], \\
& s \in \Delta_{x}(A) \leftrightarrow x \notin F_{s} \vee\left(\forall \eta \in M_{s}\right)(\exists n \in \eta)[s *\langle n\rangle \in A] .
\end{aligned}
$$


To obtain the result look at the canonical inductive operator associated with $\mathscr{X}$ and $\mathscr{H}=\left\{H_{s}: s \in \omega\right\}:$

$$
s \in \Lambda_{x}(A) \leftrightarrow x \notin H_{s} \vee\left(\forall \eta \in K_{s}\right)(\exists n \in \eta)[s *\langle n\rangle \in A] .
$$

We claim that for all $x \in X$ and $n_{0}, m_{0}, \ldots, n_{i-1}, m_{i-1}$

$$
\begin{aligned}
\left|\left(\left\langle n_{0}, \ldots, n_{i-1}\right\rangle, x\right)\right|_{\Gamma} & <\left|\left(\left\langle m_{0}, \ldots, m_{i-1}\right\rangle, x\right)\right|_{\Delta} \\
& \leftrightarrow\left\langle\left\langle n_{0}, m_{0}\right\rangle, \ldots,\left\langle n_{i-1}, m_{i-1}\right\rangle\right\rangle \in \Lambda_{x}^{\infty}
\end{aligned}
$$

and the result follows by taking $i=0$.

We shall prove the implication $(\rightarrow)$ by induction on $\left.k\left\langle n_{0}, \ldots, n_{i-1}\right\rangle, x\right)\left.\right|_{\Gamma}$. Suppose

$$
\rho=\left|\left(\left\langle n_{0}, \ldots, n_{i-1}\right\rangle, x\right)\right|_{\Gamma}<\left|\left(\left\langle m_{0}, \ldots, m_{i-1}\right\rangle, x\right)\right|_{\Delta}
$$

and assume, to the contrary, $\left\langle\left\langle n_{0}, m_{0}\right\rangle, \ldots,\left\langle n_{i-1}, m_{i-1}\right\rangle\right\rangle \notin \Lambda_{x}^{\infty}$. Then

(i) $x \in E_{\left\langle n_{0}, \ldots, n_{i-1}\right\rangle} \cup F_{\left\langle m_{0}, \ldots, m_{1-1}\right\rangle}^{c} \&\left(\exists \eta \in K_{\left\langle\left\langle n_{0}, m_{0}\right\rangle \ldots,\left\langle n_{i-1}, m_{1-1}\right\rangle\right\rangle}\right)$

$$
(\forall s \in \eta)\left[\left\langle\left\langle n_{0}, m_{0}\right\rangle, \ldots,\left\langle n_{i-1}, m_{i-1}\right\rangle\right\rangle *\langle s\rangle \notin \Lambda_{x}^{\infty}\right] .
$$

Now, $\left\langle m_{0}, \ldots, m_{i-1}\right\rangle \notin \Delta_{x}^{\rho}$ and so

$$
x \in F_{\left\langle m_{0} \ldots, m_{1-1}\right\rangle} \&\left(\exists \eta^{\prime} \in M_{\left\langle m_{0}, \ldots, m_{1-1}\right\rangle}\right)\left(\forall m \in \eta^{\prime}\right)\left[\left\langle m_{0}, \ldots, m_{i-1}, m\right\rangle \notin \Delta_{x}^{<\rho}\right] .
$$

This implies

(ii) $x \in F_{\left\langle m_{0} \ldots, m_{1-1}\right\rangle} \&$

$$
\left(\exists \eta^{\prime} \in M_{\left\langle m_{0}, \ldots, m_{1-1}\right\rangle}\right)\left(\forall m \in \eta^{\prime}\right)\left[\rho \leqslant\left|\left(\left\langle m_{0}, \ldots, m_{i-1}, m\right\rangle, x\right)\right|_{\Delta}\right] .
$$

Clearly from (i) and (ii), $x \in E_{\left\langle n_{0}, \ldots, n_{1-1}\right\rangle}$ and since $\left\langle n_{0}, \ldots, n_{i-1}\right\rangle \in \Gamma_{x}^{\rho}$,

$$
\left(\forall \eta^{\prime \prime} \in N_{\left\langle n_{0}, \ldots, n_{1-1}\right\rangle}\right)\left(\exists n \in \eta^{\prime \prime}\right)\left[\left\langle n_{0}, \ldots, n_{i-1}, n\right\rangle \in \Gamma_{x}^{<\rho}\right]
$$

which gives

(iii) $\left(\forall \eta^{\prime \prime} \in N_{\left\langle n_{0}, \ldots, n_{i-1}\right\rangle}\right)\left(\exists n \in \eta^{\prime \prime}\right)\left[\left|\left(\left\langle n_{0}, \ldots, n_{i-1}, n\right\rangle, x\right)\right|_{\Gamma}<\right.$

$$
\left.\rho=\left|\left(\left\langle n_{0}, \ldots, n_{i-1}\right\rangle, x\right)\right|_{\Gamma}\right] .
$$

Fix $\eta \in K_{\left\langle\left\langle n_{0}, m_{0}\right\rangle \ldots,\left\langle n_{1-1}, m_{t-1}\right\rangle\right)}$ to satisfy (i). Get $\eta^{\prime} \in M_{\left\langle m_{0}, \ldots, m_{1-1}\right\rangle}^{0}$ such that

$$
\left(\forall m \in \eta^{\prime}\right)\left(\exists \eta^{\prime \prime} \in N_{\left\langle n_{0}, \ldots, n_{i-1}\right\rangle}\right)\left(\forall n \in \eta^{\prime \prime}\right)[\langle n, m\rangle \in \eta] \text {. }
$$

Clearly, (ii) and (1) of $\$ 2$ yield $m^{*} \in \eta^{\prime}$ such that

$$
\rho \leqslant\left|\left(\left\langle m_{0}, \ldots, m_{i-1}, m^{*}\right\rangle, x\right)\right|_{\Delta} .
$$

By (iv) corresponding to $m^{*}$ get $\eta^{\prime \prime} \in N_{\left\langle n_{0}, \ldots, n_{1-1}\right\rangle}$ such that $\left(\forall n \in \eta^{\prime \prime}\right)\left[\left\langle n, m^{*}\right\rangle \in \eta\right]$. By (iii) get $n^{*} \in \eta^{\prime \prime}$ such that $\left.k\left\langle n_{0}, \ldots, n_{i-1}, n^{*}\right\rangle, x\right)\left.\right|_{\Gamma}<\rho$. Clearly, $\left\langle n^{*}, m^{*}\right\rangle \in \eta$ and

$$
\left|\left(\left\langle n_{0}, \ldots, n_{i-1}, n^{*}\right\rangle, x\right)\right|_{\Gamma}<\rho \leqslant\left|\left(\left\langle m_{0}, \ldots, m_{i-1}, m^{*}\right\rangle, x\right)\right|_{\Delta}
$$

and by the induction hypothesis this implies

$$
\left\langle\left\langle n_{0}, m_{0}\right\rangle, \ldots,\left\langle n_{i-1}, m_{i-1}\right\rangle,\left\langle n^{*}, m^{*}\right\rangle\right\rangle \in \Lambda_{x}^{\infty} .
$$


This clearly contradicts our choice of $\eta$. Hence $\left\langle\left\langle n_{0}, m_{0}\right\rangle, \ldots,\left\langle n_{i-1}, m_{i-1}\right\rangle\right\rangle \in \Lambda_{x}^{\infty}$.

To prove the other implication, set

$$
\begin{aligned}
A^{*}= & \left\{\left\langle\left\langle n_{0}, m_{0}\right\rangle, \ldots,\left\langle n_{i-1}, m_{i-1}\right\rangle\right\rangle:\right. \\
& \left.\left|\left(\left\langle n_{i}, \ldots, n_{i-1}\right\rangle, x\right)\right|_{\Gamma}<\left|\left(\left\langle m_{0}, \ldots, m_{i-1}\right\rangle, x\right)\right|_{\Delta}\right\} \\
& \cup\left\{t: t \text { is not of the form }\left\langle\left\langle n_{0}, m_{0}\right\rangle, \ldots,\left\langle m_{i-1}, m_{i-1}\right\rangle\right\rangle\right\} .
\end{aligned}
$$

We shall show that $\Lambda_{x}\left(A^{*}\right) \subseteq A^{*}$, from which it will follow that $\Lambda_{x}^{\infty} \subseteq A^{*}$.

So let

$$
\left\langle\left\langle n_{0}, m_{0}\right\rangle, \ldots,\left\langle n_{i-1}, m_{i-1}\right\rangle\right\rangle \in \Lambda_{x}\left(A^{*}\right) .
$$

We will have to show that

$$
\left|\left(\left\langle n_{0}, \ldots, n_{i-1}\right\rangle, x\right)\right|_{\Gamma}<\left|\left(\left\langle m_{0}, \ldots, m_{i-1}\right\rangle, x\right)\right|_{\Delta} .
$$

Assume to the contrary that

$$
\left|\left(\left\langle m_{0}, \ldots, m_{i-1}\right\rangle, x\right)\right|_{\Delta} \leqslant\left|\left(\left\langle n_{0}, \ldots, n_{i-1}\right\rangle, x\right)\right|_{\Gamma} .
$$

From (v) we have

$$
\begin{aligned}
& x \in E_{\left\langle n_{0}, \ldots, n_{t-1}\right\rangle}^{c} \cap F_{\left\langle m_{0}, \ldots, m_{t-1}\right\rangle} \vee\left(\forall \eta \in K_{\left\langle\left\langle n_{0}, m_{0}\right\rangle \ldots\left\langle n_{t-1}, m_{t-1}\right\rangle\right\rangle}\right)(\exists s \in \eta) \\
& {\left[\left\langle\left\langle n_{0}, m_{0}\right\rangle, \ldots,\left\langle n_{i-1}, m_{i-1}\right\rangle, s\right\rangle \in A^{*}\right] . }
\end{aligned}
$$

If $x \in E_{\left\langle n_{0}, \ldots, n_{t-1}\right\rangle}^{c} \cap F_{\left\langle m_{0}, \ldots, m_{t-1}\right\rangle}$, then $\left|\left(\left\langle n_{0}, \ldots, n_{i-1}\right\rangle, x\right)\right|_{\Gamma}=0$ and

$$
\left|\left(\left\langle m_{0}, \ldots, m_{i-1}\right\rangle, x\right)\right|_{\Delta}>0
$$

and we are done. So assume

(vi) $x \in\left(E_{\left\langle n_{0}, \ldots, n_{1-1}\right\rangle} \cup F_{\left\langle m_{0}, \ldots, m_{1-1}\right\rangle}^{c}\right) \&\left(\forall \eta \in K_{\left\langle\left\langle n_{0}, m_{0}\right\rangle \ldots\left\langle n_{1-1}, m_{1-1}\right\rangle\right)}\right)(\exists s \in \eta)$

$$
\left[\left\langle\left\langle n_{0}, m_{0}\right\rangle, \ldots,\left\langle n_{i-1}, m_{i-1}\right\rangle, s\right\rangle \in A^{*}\right] \text {. }
$$

If $x \in F_{\left\langle m_{0}, \ldots, m_{t-1}\right\rangle}^{c}$, then by regularity $x \in F_{\left\langle m_{0} \ldots, m_{t-1}, m\right\rangle}^{c}$ for all $m$, and hence $\left.\left.(\forall m)\left[k\left\langle m_{0}, \ldots, m_{i-1}, m\right\rangle, x\right)\right|_{\Delta}=0\right]$. But this is not possible by (vi). Therefore,

$$
\begin{aligned}
& x \in\left(E_{\left\langle n_{0}, \ldots, n_{1-1}\right\rangle}\right.\left.\cap F_{\left\langle m_{0}, \ldots, m_{1-1}\right\rangle}\right) \\
& \&\left(\forall \eta \in K_{\left\langle\left\langle n_{0}, m_{0}\right\rangle, \ldots\left\langle n_{1-1}, m_{1-1}\right\rangle\right\rangle}\right)(\exists s \in \eta) \\
& {\left[\left\langle\left\langle n_{0}, m_{0}\right\rangle, \ldots,\left\langle n_{i-1}, m_{i-1}\right\rangle, s\right\rangle \in A^{*}\right] . }
\end{aligned}
$$

Case 1. $\left|\left(\left\langle n_{0}, \ldots, n_{i-1}\right\rangle, x\right)\right|_{\Gamma}=\omega_{1}$. In this case $\left\langle n_{0}, \ldots, n_{i-1}\right\rangle \notin \Gamma_{x}^{\infty}$ and hence

$$
\left(\exists \eta^{\prime \prime} \in N_{\left\langle n_{0} \ldots, n_{1-1}\right\rangle}\right)\left(\forall n \in \eta^{\prime \prime}\right)\left[\left|\left(\left\langle n_{0}, \ldots, n_{i-1}, n\right\rangle, x\right)\right|_{\Gamma}=\omega_{1}\right] .
$$

Fix such an $\eta^{\prime \prime} \in N_{\left\langle n_{0} \ldots, n_{1-1}\right\rangle}$. Pick any $\eta^{\prime} \in M_{\left\langle m_{0} \ldots, m_{1-1}\right\rangle}^{0}$ and put

$$
\eta^{*}=\left\{\langle n, m\rangle: n \in \eta^{\prime \prime} \& m \in \eta^{\prime}\right\} \text {. }
$$


Clearly $\eta^{*} \in K_{\left\langle\left\langle n_{0}, m_{0}\right\rangle, \ldots\left\langle n_{t-1}, m_{i-1}\right\rangle\right\rangle}$ and, moreover,

$$
\left(\forall\langle n, m\rangle \in \eta^{*}\right)\left[\left|\left(\left\langle m_{0}, \ldots, m_{i-1}, m\right\rangle, x\right)\right|_{\Delta} \leqslant \omega_{1}=\left|\left(\left\langle n_{0}, \ldots, n_{i-1}, n\right\rangle, x\right)\right|_{\Gamma}\right] .
$$

This contradicts (vii).

Case 2. $\left.K\left\langle n_{0}, \ldots, n_{i-1}\right\rangle, x\right)\left.\right|_{\Gamma}=\rho<\omega_{1}$. Here we have, by our assumption, $\left\langle m_{0}, \ldots, m_{i-1}\right\rangle \in \Delta_{x}^{\rho}$ and since $\left.x \in F_{\left\langle m_{0} \ldots m_{1-1}\right\rangle}\right\rangle$

$$
\left(\forall \eta^{\prime} \in M_{\left\langle m_{0}, \ldots, m_{l-1}\right\rangle}\right)\left(\exists m \in \eta^{\prime}\right)\left[\left|\left(\left\langle m_{0}, \ldots, m_{i-1}, m\right\rangle, x\right)\right|_{\Delta}<\rho\right]
$$

which implies by (2) that

$$
\begin{aligned}
& \left(\exists \eta^{\prime} \in M_{\left\langle m_{0}, \ldots, m_{t-1}\right\rangle}^{0}\right)\left(\forall m \in \eta^{\prime}\right)\left[\left|\left(\left\langle m_{0}, \ldots, m_{i-1}, m\right\rangle, x\right)\right|_{\Delta}<\rho\right] \text { hence, } \\
& \left(\exists \eta^{\prime} \in M_{\left\langle m_{0}, \ldots, m_{\iota-1}\right\rangle}^{0}\right)\left(\forall m \in \eta^{\prime}\right)\left[\left\langle n_{0}, \ldots, n_{i-1}\right\rangle \notin \Gamma_{x}^{\left|\left(\left\langle m_{0} \ldots, m_{t-1}, m\right\rangle ; x\right)\right|_{\perp}}\right] .
\end{aligned}
$$

Consequently,

$$
\begin{gathered}
\left(\exists \eta^{\prime} \in M_{\left\langle m_{0} \ldots, m_{i-1}\right\rangle}^{0}\right)\left(\forall m \in \eta^{\prime}\right)\left(\exists \eta^{\prime \prime} \in N_{\left\langle n_{0} \ldots, n_{i-1}\right\rangle}\right)\left(\forall n \in \eta^{\prime \prime}\right) \\
{\left[\left|\left(\left\langle m_{0}, \ldots, m_{i-1}, m\right\rangle, x\right)\right|_{\Delta} \leqslant\left|\left(\left\langle n_{0}, \ldots, n_{i-1}, n\right\rangle, x\right)\right|_{\Gamma}\right] .}
\end{gathered}
$$

This clearly contradicts (vii). Thus in either case we have a contradiction and so $\Lambda_{x}^{\infty}\left(A^{*}\right) \subseteq A^{*}$. Thus $\Lambda_{x}^{\infty} \subseteq A^{*}$. This proves the other implication of (8).

The following trick is due to Lyapunov.

LEMMA 4.3 (INCREASING THE INDEX BY 1). Let $\Gamma$ be the canonical inductive operator associated with $\mathscr{E}=\left\{E_{p}: p \in \omega\right\}$ and $\mathscr{N}=\left\{N_{k}: k \in \omega\right\}$. Define

$$
\begin{aligned}
& E_{\langle\rangle}^{*}=X \\
& E_{s}^{*}=\left\{\begin{array}{l}
E_{\left\langle n_{1}, \ldots, n_{k}\right\rangle} \quad \text { if } s=\left\langle 1, n_{1}, \ldots, n_{k}\right\rangle, \\
\varnothing \quad \text { otherwise. }
\end{array}\right.
\end{aligned}
$$

Put

$$
\begin{gathered}
N_{\zeta\rangle}^{*}=\{\{1\}\} ; \\
N_{s}^{*}=\left\{\begin{array}{l}
N_{\left\langle n_{1}, \ldots, n_{k}\right\rangle} \text { if } s=\left\langle 1, n_{1}, \ldots, n_{k}\right\rangle, \\
\{\langle n\}: n \in \omega\} \quad \text { otherwise. }
\end{array}\right.
\end{gathered}
$$

If $\mathscr{N}^{*}=\left\{N_{k}^{*}: k \in \omega\right\}$, then

$$
\mathscr{R}_{\mathscr{N}^{*}}\left(\left\{E_{s}^{*}: s \in \omega\right\}\right)=\mathscr{R}_{\mathscr{N}}\left(\left\{E_{s}: s \in \omega\right\}\right)
$$

and $|(e, x)|_{\Gamma^{*}}=|(e, x)|_{\Gamma}+1$, where $\Gamma^{*}$ is the inductive operator associated with

$$
\mathscr{E}^{*}=\left\{E_{s}^{*}: s \in \omega\right\} \text { and } \mathscr{N}^{*} \text {. }
$$

Proof. We shall prove by induction on $\rho$ that

$$
\left\langle 1, n_{1}, \ldots, n_{k}\right\rangle \in \Gamma_{x}^{* \rho} \leftrightarrow\left\langle n_{1}, \ldots, n_{k}\right\rangle \in \Gamma_{x}^{\rho} .
$$


Now,

$$
\begin{aligned}
&\left\langle 1, n_{1}, \ldots, n_{k}\right\rangle \in \Gamma_{x}^{* \rho} \leftrightarrow x \notin E_{\left\langle 1, n_{1}, \ldots, n_{k}\right\rangle}^{*} \vee \\
&\left(\forall \eta \in N_{\left\langle 1, n_{1}, \ldots, n_{k}\right\rangle}^{*}\right)(\exists n \in \eta)\left[\left\langle 1, n_{1}, \ldots, n_{k}, n\right\rangle \in \Gamma_{x}^{*<\rho}\right] \\
& \leftrightarrow x \notin E_{\left\langle n_{1}, \ldots, n_{h}\right\rangle} \vee \\
&\left(\forall \eta \in N_{\left\langle n_{1}, \ldots, n_{k}\right\rangle}\right)(\exists n \in \eta)\left[\left\langle n_{1}, \ldots, n_{k}, n\right\rangle \in \Gamma_{x}^{<\rho}\right] \\
& \leftrightarrow\left\langle n_{1}, \ldots, n_{k}\right\rangle \in \Gamma_{x}^{\rho} . \\
& \therefore e \in \Gamma_{x}^{* \rho+1} \leftrightarrow\left(\forall \eta \in N_{e}^{*}\right)(\exists n \in \eta)\left[\langle n\rangle \in \Gamma_{x}^{* \rho}\right] \\
& \leftrightarrow\langle 1\rangle \in \Gamma_{x}^{* \rho} \\
& \leftrightarrow e \in \Gamma_{x}^{\rho} .
\end{aligned}
$$

Hence $\mathscr{R}_{\mathscr{N}^{*}}\left(\left\{E_{s}^{*}: s \in \omega\right\}\right)=\mathscr{R}_{\mathscr{N}}\left(\left\{E_{s}: s \in \omega\right\}\right)$ and $|(e, x)|_{\Gamma^{*}}=|(e, x)|_{\Gamma}+1$.

The following lemma follows from above. One has only to observe that for any positive analytical operation $\Phi, R \Phi$ is normal, $R \Phi^{0} \Phi \sim R \Phi \Phi^{0}$ and if

$$
\mathscr{N}=\left\{N_{s}: s \in \omega\right\}
$$

is a family of bases such that $\Phi \geqslant \Phi_{N_{s}}$ for each $s$, then $R \Phi \geqslant \mathscr{R}_{\mathscr{N}}$.

LEMMA 4.4. Let $\Phi \geqslant \cup$ be a positive analytical operation and $\mathscr{N}=\left\{N_{s}: s \in \omega\right\}$, $\mathscr{M}=\left\{M_{s}: s \in \omega\right\}$ be two families of bases such that for each $s, \Phi^{*}$ subsumes $\Phi_{N_{s}}$ and $\Phi_{M_{s}^{0} .}$ Suppose $\left\{E_{s}: s \in \omega\right\}$ is a family of sets in $\Sigma_{1}^{\Phi^{*}}$ and $\left\{F_{s}: s \in \omega\right\}$ a regular family in $\Pi_{1}^{\Phi^{*}}$. Let $\Gamma$ be the canonical inductive operator associated with $\mathscr{E}=\left\{E_{s}: s \in \omega\right\}$ and $\mathscr{N}$, and $\Delta$ that associated with $\mathscr{F}=\left\{F_{s}: s \in \omega\right\}$ and $\mathscr{M}$. Put $\beta_{1}(x)=|(e, x)|_{\Gamma}$ and $\beta_{2}(x)=|(e, x)|_{\Delta}$. Then

(a) $\left\{x: \beta_{1}(x)<\beta_{2}(x)\right\} \in \Pi_{1}^{\Phi^{*}}$,

(b) $\left\{x: \beta_{1}(x)<\omega_{1} \& \beta_{1}(x) \leqslant \beta_{2}(x)\right\} \in \Pi_{1}^{\Phi^{*}}$.

Proof. The first assertion follows from the comparison of indices lemma and the observations made above. The second assertion follows from the first by increasing the index of $\Delta$ by 1 .

By slightly modifying the inductive operator $\Lambda$ in the proof of Lemma 4.2 , one can obtain the following

Corollary 4.5. Let $\Gamma, \Delta, \Phi$ be as in 4.4. Then

$$
\left\{(s, t, x):|(s, x)|_{\mathrm{r}}<|(t, x)|_{\Delta}\right\} \in \Pi_{1}^{\Phi^{*}} .
$$

THEOREM 4.6. For any positive analytical operation $\Phi \geqslant U, \Pi_{1}^{\Phi^{*}}$ has the pre-wellordering property.

Proof. Let $E \in \Pi_{1}^{\Phi^{*}}$ and suppose

$$
E^{c}=\Phi^{*}\left(\left\{A_{s}: s \in \omega\right\}\right) \text { with }\left\{A_{s}\right\} \text { clopen and regular. }
$$

Let $\beta(x)$ be the norm on $E$ induced by the canonical inductive operator. Let $N$ be the canonical base for $\Phi$ and put $\mathscr{N}=\left\{N N^{0}\right\}, \mathscr{H}=\left\{N N^{0}\right\}$. For each $s$, set

$$
E_{s}=A_{s} \times X, \quad F_{s}=X \times A_{s}
$$


and let $\beta_{1}(x, y), \beta_{2}(x, y)$ be the norms induced by the inductive operators associated with $\left\{E_{s}: s \in \omega\right\}, \mathscr{N}$ and $\left\{F_{s}: s \in \omega\right\}, \mathscr{M}$. Since all the hypotheses of Lemma 4.4 are satisfied, the sets $\left\{(x, y): \beta_{1}(x, y)<\beta_{2}(x, y)\right\}$ and

$$
\left\{(x, y): \beta_{1}(x, y)<\omega_{1} \& \beta_{1}(x, y) \leqslant \beta_{2}(x, y)\right\}
$$

are in $\Pi_{1}^{\Phi^{*}}$. But

$$
\left\{(x, y): \beta_{1}(x, y)<\beta_{2}(x, y)\right\}=\{(x, y): \beta(x)<\beta(y)\}
$$

and

$$
\begin{aligned}
\left\{(x, y): \beta_{1}(x, y)\right. & \left.<\omega_{1} \& \beta_{1}(x, y) \leqslant \beta_{2}(x, y)\right\} \\
& =\left\{(x, y): \beta(x)<\omega_{1} \& \beta(x) \leqslant \beta(y)\right\} .
\end{aligned}
$$

Consequently, $\Pi_{1}^{\Phi^{*}}$ is normed.

COROLLARY 4.7. For each $\rho<\omega_{1}, c \mathscr{R}^{\rho}$ has the pre-well-ordering property.

5. Complexity of winning strategies and the transfer property of the $R$-operator.

THEOREM 5.1. Let $\Phi$ be a positive analytical operation which subsumes both (countable) $\cup$ and $\cap$. Let $\nabla$ be the $\sigma$-field generated by $\Sigma_{1}^{\Phi^{*}}$. Let $E \in \Sigma_{1}^{\phi^{*}}$ be such that

(i) $x \in E \leftrightarrow\left(\exists \eta_{0} \in N N^{0}\right)\left(\forall n_{0} \in \eta_{0}\right)\left(\exists \eta_{1} \in N N^{0}\right)\left(\forall n_{1} \in \eta_{1}\right) \cdots$

$$
\cdots(\forall k)\left[x \in E_{\left\langle n_{0} \ldots, n_{k-1}\right\rangle}\right] \text {, }
$$

$N$ being the canonical base for $\Phi$. Then, there is a $\nabla$-measurable function $x \rightarrow \sigma_{x}$ such that $\sigma_{x}$ is a winning strategy for the player $\exists$, whenever $x \in E$.

Proof. Let $\Gamma$ be the canonical inductive operator associated with $N N^{0}$ and $\left\{E_{s}: s \in \omega\right\}$ and put $N N^{0}=M$. Define

$$
\beta(s, x)= \begin{cases}\text { least } \rho \text { such that } s \in \Gamma_{x}^{\rho} & \text { if } s \in \Gamma_{x}^{\infty}, \\ \omega_{1} & \text { otherwise. }\end{cases}
$$

Now suppose $x \in E$. So $\exists$ wins the game (i).

If $\eta_{0}, \eta_{1}, \ldots, \eta_{k-1}$ and $n_{0}, n_{1}, \ldots, n_{k-1}$ are the first $k$ relevant moves of $\exists$ and $\forall$, notice that $\exists$ goes on to win the game (i), i.e., he is in a winning position iff $\left\langle n_{0}, \ldots, n_{k-1}\right\rangle \notin \Gamma_{x}^{\infty}$ i.e., iff $\beta\left(\left\langle n_{0}, \ldots, n_{k-1}\right\rangle, x\right)=\omega_{1}$. In such a case, $\exists$ has to play an $\eta \in M$ such that $(\forall n \in \eta)\left[\beta\left(\left\langle n_{0}, \ldots, n_{k-1}, n\right\rangle, x\right)=\omega_{1}\right]$. We, therefore, define for each $x$, the strategy $\sigma_{x}$ for $\exists$ as follows:

$$
p \in \sigma_{x}(s) \leftrightarrow \beta(s, x) \leqslant \beta(s *\langle p\rangle, x) .
$$

Clearly by 4.5 , the map $x \rightarrow \sigma_{x}$ is $\nabla$-measurable. We shall now show that if $x \in E$, then $\sigma_{x}$ is a winning strategy for $\exists$ in the game (i). Suppose $\eta_{0}, n_{0}, \eta_{1}, n_{1}, \ldots, \eta_{k-1}$, $n_{k-1}$ are the first $k$ moves of $\exists$ and $\forall$ and assume that $\exists$ has not yet lost the game i.e. he is in a winning position. Consequently, we have $\left(\beta\left(\left\langle n_{0}, n_{1}, \ldots, n_{k-1}\right\rangle, x\right)=\omega_{1}\right.$ and hence $\left\langle n_{0}, \ldots, n_{k-1}\right\rangle \notin \Gamma_{x}^{\infty}$. Therefore,

$$
(\exists \eta \in M)(\forall n \in \eta)\left[\beta\left(\left\langle n_{0}, n_{1}, \ldots, n_{k-1}, n\right\rangle, x\right)=\omega_{1}\right] \text {. }
$$


By definition,

$$
\begin{aligned}
\sigma_{x}\left(\left\langle n_{0}, \ldots, n_{k-1}\right\rangle\right) & =\left\{p: \beta\left(\left\langle n_{0}, \ldots, n_{k-1}\right\rangle, x\right) \leqslant \beta\left(\left\langle n_{0}, \ldots, n_{k-1}, p\right\rangle, x\right)\right\} \\
& =\left\{p: \beta\left(\left\langle n_{0}, \ldots, n_{k-1}, p\right\rangle, x\right)=\omega_{1}\right\} .
\end{aligned}
$$

Hence, by (ii) and the completeness of $M$,

$$
\sigma_{x}\left(\left\langle n_{0}, \ldots, n_{k-1}\right\rangle\right)=\eta \in M,
$$

and moreover, $\forall p \in \eta, \beta\left(\left\langle n_{0}, \ldots, n_{k-1}, p\right\rangle, x\right)=\omega_{1}$, so that $\exists$ is still in a winning position.

REMARK. Notice that

(iii)

$$
\begin{aligned}
x \notin E \leftrightarrow\left(\forall \eta_{0} \in M\right)\left(\exists n_{0} \in \eta_{0}\right)\left(\forall \eta_{1} \in M\right)\left(\exists n_{1} \in \eta_{1}\right) \cdots(\exists k)\left[x \notin E_{\left\langle n_{0} \ldots, n_{k-1}\right\rangle}\right] \\
\leftrightarrow\left(\exists \eta_{0} \in M^{0}\right)\left(\forall n_{0} \in \eta_{0}\right) \cdots(\exists k)\left[x \notin E_{\left\langle n_{0} \ldots, n_{k-1}\right\rangle}\right] .
\end{aligned}
$$

Here also we can have a definable winning strategy for $\exists$ whenever $x \notin E$. Unlike the game (i), here $\exists$ has to play such that at each stage the value of $\beta$ is decreased. The following will give a $\nabla$-measurable winning strategy for $\exists$ in the game (iii) whenever $x \notin E$ :

$$
p \in \sigma_{x}(s) \leftrightarrow x \notin E_{s} \vee(\beta(s *\langle p\rangle, x)<\beta(s, x)) .
$$

Definition 5.2. A set $E \subseteq X \times Y$ is said to be normal if for each $x \in X$, $E^{x}=\{y:(x, y) \in E\}$ has the Baire property. If $E$ is normal and $U \subseteq Y$ is open, then define

$$
E^{* U}=\left\{x \in X: E^{x} \text { is comeager in } U\right\} .
$$

If $U=Y$, we write $E^{*}$ instead of $E^{* U}$.

LEMMA 5.3. Let $\Phi$ be a positive analytical operation which preserves the Baire property and let $E=\Phi^{*}\left(\left\{E_{s}: s \in \omega\right\}\right)$, with each $E_{s} \subseteq \omega^{\omega} \times \omega^{\omega}$ normal. Define $E_{s}^{\mu}$ and $T^{\mu}$ as in 3.6. Then for any $s \in \omega$,

$$
E^{\cdot \Sigma(s)}=\bigcap_{\mu<\omega_{1}}\left[E_{e}^{\mu}\right]^{* \Sigma(s)}=\bigcup_{\mu<\omega_{1}}\left[E_{e}^{\mu}-T^{\mu}\right]^{* \Sigma(s)} .
$$

Proof. As in Theorem 3.8, one can easily check that $E_{s}^{\mu}$ and $T^{\mu}$ are normal for each $\mu$. Since $E=\bigcap_{\mu<\omega_{1}} E_{e}^{\mu}$, it follows that

$$
E^{* \Sigma(s)} \subseteq \bigcap_{\mu<\omega_{1}}\left[E_{e}^{\mu}\right]^{* \Sigma(s)}
$$

Next, suppose $x \in\left[E_{e}^{\mu}\right]^{* \Sigma(s)}$ for all $\mu<\omega_{1}$. For each $p \in \omega,\left\{\left(E_{p}^{\mu}\right)^{x}: \mu<\omega_{1}\right\}$ is a decreasing sequence of sets with Baire property. Hence by the countable chain condition, $\exists \beta(p)<\omega_{1}$ such that

$$
(\forall \rho>\beta(p))\left[\left(E_{p}^{\beta(p)}-E_{p}^{\rho}\right)^{x} \text { is meager }\right] .
$$

Choose $\rho_{0}$ such that $\beta(p)<\rho_{0}<\omega_{1}$, for all $p$. Then $(\forall p)\left[\left(E_{p}^{\rho_{0}}-E_{p}^{\rho_{0}+1}\right)^{x}\right.$ is meager] and hence $\left(T^{\rho_{0}}\right)^{x}$ is meager. Since $x \in\left[E_{e}^{\rho_{0}}\right]^{* \Sigma(s)},\left(E_{e}^{\rho_{0}}\right)^{x}$ is comeager in $\Sigma(s)$. Therefore, $\left(E_{e}^{\rho_{0}}\right)^{x}-\left(T^{\rho_{0}}\right)^{x}$ is comeager in $\Sigma(s)$ and so $x \in\left[E_{e}^{\rho_{0}}-T^{\rho_{0}}\right]^{* \Sigma(s)}$. 
Thus,

(ii)

$$
\bigcap_{\mu<\omega_{1}}\left[E_{e}^{\mu}\right]^{* \Sigma(s)} \subseteq \bigcup_{\mu<\omega_{1}}\left[E_{e}^{\mu}-T^{\mu}\right]^{* \Sigma(s)}
$$

Finally, since $\left(E_{e}^{\mu}-T^{\mu}\right) \subseteq E$ for each $\mu$,

$$
\bigcup_{\mu<\omega_{1}}\left[E_{e}^{\mu}-T^{\mu}\right]^{* \Sigma(s)} \subseteq E^{* \Sigma(s)} .
$$

The result now follows from (i)-(iii).

TRANSFER THEOREM 5.4. Let $\Phi$ and $\Psi$ be two positive analytical operations such that $\Phi$ preserves the Baire property and $\Psi$ is normal and subsumes both (countable) $\cup$ and $\cap$. Suppose, moreover, that there are functions $f$ and $g$ such that for any normal family $\left\{E_{p}: p \in \omega\right\}$ of subsets of $\omega^{\omega} \times \omega^{\omega}$ with $E=\Phi\left(\left\{E_{p}: p \in \omega\right\}\right)$,

$$
E^{* \Sigma(s)}=\Psi\left(\left\{E_{f(p)} \Sigma^{\Sigma(\hat{s} g(p))}: p \in \omega\right\}\right) .
$$

Then for any normal family $\left\{F_{p}: p \in \omega\right\}$ of subsets of $\omega^{\omega} \times \omega^{\omega}$,

(a) $F=\Phi^{0}\left(\left\{F_{p}: p \in \omega\right\}\right)$ implies that

$$
F^{* \Sigma(s)}=\Psi^{0}\left(\left\{F_{\gamma(p)}^{* \Sigma(\hat{\delta} \delta(p))} ; p \in \omega\right\}\right)
$$

for suitable functions $\gamma$ and $\delta$ (independent of the family $\left\{F_{p}\right\}$ ).

(b) $F=\Phi \cdot \Phi^{0}\left(\left\{F_{p}: p \in \omega\right\}\right)$ implies

$$
F^{* \Sigma(s)}=\Psi \Psi^{0}\left(\left\{F_{\alpha(p)}^{* \Sigma(\hat{s} \beta(p))} ; p \in \omega\right\}\right)
$$

where

$$
\begin{gathered}
\alpha(s)= \begin{cases}\langle f(n), \gamma(m)\rangle & \text { if } s=\langle n, m\rangle, \\
\text { arbitrary } & \text { otherwise; }\end{cases} \\
\beta(s)= \begin{cases}g(n) \hat{\delta}(m) & \text { if } s=\langle n, m\rangle, \\
\text { arbitrary } & \text { otherwise. }\end{cases}
\end{gathered}
$$

(c) $F=\Phi^{*}\left(\left\{F_{p}: p \in \omega\right\}\right)$ implies

$$
F^{* \Sigma(s)}=\Psi^{*}\left(\left\{F_{\tilde{f}(p)}^{* \Sigma(\hat{s} \tilde{g}(p))}: p \in \omega\right\}\right),
$$

where

$$
\begin{gathered}
\tilde{f}(s)=\left\{\begin{array}{l}
\left\langle\alpha\left(n_{0}\right), \ldots, \alpha\left(n_{k-1}\right)\right\rangle \\
\text { arbitrary } \text { otherwise; }
\end{array} \text { if } s=\left\langle n_{0}, \ldots, n_{k-1}\right\rangle,\right. \\
\tilde{g}(s)=\left\{\begin{array}{l}
\beta\left(n_{0}\right) \hat{\beta}\left(n_{1}\right) \ldots \hat{\beta}\left(n_{k-1}\right) \text { if } s=\left\langle n_{0}, \ldots, n_{k-1}\right\rangle, \\
\text { arbitrary } \text { otherwise. }
\end{array}\right.
\end{gathered}
$$

Proof. Let $N$ and $M$ be the canonical bases for $\Phi$ and $\Psi$, respectively.

(a) Set $G=F^{c}$ and $G_{p}=F_{p}^{c}$ for each $p$.

Then $G=\Phi\left(\left\{G_{p}: p \in \omega\right\}\right)$; and since $\Phi$ preserves the Baire property, each $G^{x}$ has 
the Baire property. Therefore,

$G^{x}$ is nonmeager in $\Sigma(s)$

$\leftrightarrow(\exists u)\left[G^{x}\right.$ is comeager in $\left.\Sigma(\hat{s u})\right]$

$\leftrightarrow(\exists u)(\exists \eta \in M)(\forall m \in \eta)\left[G_{f(m)}^{x}\right.$ is comeager in $\left.\Sigma(\hat{s u} g(m))\right]$ by hypothesis

$\leftrightarrow(\exists u)(\exists \eta \in M)(\forall m \in \eta)(\forall v)\left[G_{f(m)}^{x}\right.$ is nonmeager in $\left.\Sigma(\hat{s} \hat{u} g(m) \hat{v})\right]$

$\leftrightarrow(\exists \eta \in M)(\forall m \in \eta)\left[G_{\gamma(m)}^{x}\right.$ is nonmeager in $\left.\Sigma(\hat{s} \delta(m))\right]$,

for some functions $\gamma$ and $\delta$, as $\Phi_{M} \geqslant \cap, \cup$ and is normal. Hence,

$F^{x}$ is comeager in $\Sigma(s) \leftrightarrow G^{x}$ is meager in $\Sigma(s)$

$$
\begin{aligned}
& \leftrightarrow(\forall \eta \in M)(\exists m \in \eta)\left[G_{\gamma(m)}^{x} \text { is meager in } \Sigma(\hat{s} \delta(m))\right] \\
& \leftrightarrow(\forall \eta \in M)(\exists m \in \eta)\left[F_{\gamma(m)}^{x} \text { is comeager in } \Sigma(\hat{s} \delta(m))\right] \\
& \leftrightarrow\left(\exists \eta \in M^{0}\right)(\forall m \in \eta)\left[F_{\gamma(m)}^{x} \text { is comeager in } \Sigma(\hat{s} \delta(m))\right] .
\end{aligned}
$$

This proves (a).

To prove (b), observe that

$F^{x}$ is comeager in $\Sigma(s)$

$\leftrightarrow\left[\Phi\left(\left\{\Phi^{0}\left(\left\{F_{\langle p, q\rangle}: q \in \omega\right\}\right): p \in \omega\right\}\right)\right]^{x}$ is comeager in $\Sigma(s)$

$\leftrightarrow(\exists \eta \in M)(\forall n \in \eta)\left[\left(\Phi^{0}\left(\left\{F_{\langle f(n), q\rangle}: q \in \omega\right\}\right)\right)^{x}\right.$ is comeager in $\left.\Sigma(\hat{s g}(n))\right]$

(by hypothesis),

$\leftrightarrow(\exists \eta \in M)(\forall n \in \eta)\left(\exists \xi \in M^{0}\right)(\forall m \in \xi)$

$$
\left[F_{\langle f(n), \gamma(m)\rangle}^{x} \text { is comeager in } \Sigma(\hat{s g}(n) \hat{\delta}(m))\right] \quad(\text { by }(\mathrm{a})) \text {. }
$$

Setting $\alpha(\langle n, m\rangle)=\langle f(n), \gamma(m)\rangle, \beta(\langle n, m\rangle)=g(n) \hat{\delta}(m)$, we get (b).

(c) Since $F=\Phi^{*}\left(\left\{F_{p}: p \in \omega\right\}\right)$, the canonical inductive operator $\Gamma$ is given by

$p \in \Gamma_{z}(A) \leftrightarrow z \notin F_{p} \vee(\forall \eta \in N)(\exists n \in \eta)(\exists \xi \in N)(\forall m \in \xi)$

$$
[p *\langle\langle n, m\rangle\rangle \in A] ; z \in \omega^{\omega} \times \omega^{\omega} \text {. }
$$

Define $z \in F_{p}^{\mu} \leftrightarrow p \notin \Gamma_{z}^{\mu}$. Then by Lemma 5.3,

$$
F^{\cdot \Sigma(s)}=\bigcap_{\mu<\omega_{1}}\left[F_{e}^{\mu}\right]^{\cdot \Sigma(s)} .
$$

Define a set relation $\Delta$ operative on $\omega$ as follows:

$t \in \Delta_{x}(A) \leftrightarrow F_{(t)_{0}}^{x}$ is not comeager in $\Sigma\left((t)_{1}\right) \vee$

$$
(\forall \eta \in M)(\exists n \in \eta)(\exists \xi \in M)(\forall m \in \xi)
$$

$$
\left[\left\langle(t)_{0} \hat{\langle}\langle\langle f(n), \gamma(m)\rangle\rangle,(t)_{1} \hat{g}(n) \hat{\delta}(m)\right\rangle \in A\right] .
$$

We shall show that

$$
x \notin F^{* \Sigma(s)} \leftrightarrow\langle e, s\rangle \in \Delta_{x}^{\infty} .
$$


To prove (ii) we shall show by induction on $\mu$ that

$$
x \notin\left[F_{t}^{\mu}\right]^{\bullet \Sigma(s)} \leftrightarrow\langle t, s\rangle \in \Delta_{x}^{\mu} .
$$

This is clearly true for $\mu=0$, so assume $\mu>0$. Now, $x \notin\left[F_{t}^{\mu}\right]^{* \Sigma(s)} \leftrightarrow\left(F_{t}^{\mu}\right)^{x}$ is not comeager in $\Sigma(s)$

$\leftrightarrow F_{t}^{x}$ is not comeager in $\Sigma(s) \vee\left[\Phi_{N N^{0}}\left(\left\{\bigcap_{\lambda<\mu} F_{i\langle p\rangle}^{\lambda}: p \in \omega\right\}\right)\right]^{x}$

is not comeager in $\Sigma(s)$

$\leftrightarrow F_{t}^{x}$ is not comeager in $\Sigma(s) \vee(\forall \eta \in M)(\exists n \in \eta)(\exists \xi \in M)(\forall m \in \xi)$

$$
\left.\left[\left(\bigcap_{\lambda<\mu} F_{t}^{\lambda}\langle\langle f(n), \gamma(m)\rangle\rangle\right)\right)^{x} \text { is not comeager in } \Sigma(\hat{s} g(n) \hat{\delta}(m))\right] \quad \text { (by (b)) }
$$

$\leftrightarrow F_{t}^{x}$ is not comeager in $\Sigma(s) \vee(\forall \eta \in M)(\exists n \in \eta)(\exists \xi \in M)(\forall m \in \xi)$

$$
(\exists \lambda<\mu)\left[\left(F_{t}^{\lambda}\langle\langle f(n), \gamma(m)\rangle\rangle\right)^{x} \text { is not comeager in } \Sigma(\hat{s g}(n) \hat{\delta}(m))\right]
$$

$\leftrightarrow F_{t}^{x}$ is not comeager in $\Sigma(s) \vee(\forall \eta \in M)(\exists n \in \eta)(\exists \xi \in M)(\forall m \in \xi)$

$$
(\exists \lambda<\mu)\left[\langle\hat{t}\langle\langle f(n), \gamma(m)\rangle\rangle, \hat{s} g(n) \hat{\delta}(m)\rangle \in \Delta_{x}^{\lambda}\right]
$$

by the induction hypothesis

$\leftrightarrow\langle t, s\rangle \in \Delta_{s}^{\mu}$.

Hence, putting $t=\langle\rangle$ and using (i), we obtain (ii). Therefore,

$F^{x}$ is comeager in $\Sigma(s) \leftrightarrow x \in F^{* \Sigma(s)} \leftrightarrow\langle e, s\rangle \notin \Delta_{x}^{\infty}$ by (ii)

$$
\leftrightarrow\left(\exists \eta_{0} \in M\right)\left(\forall n_{0} \in \eta_{0}\right)\left(\forall \xi_{0} \in M\right)\left(\exists m_{0} \in \xi_{0}\right) \cdots
$$

$(\forall k)\left[F^{x}\left\langle\left\langle f\left(n_{0}\right), \gamma\left(m_{0}\right)\right\rangle \ldots,\left\langle f\left(n_{k-1}\right), \gamma\left(m_{k-1}\right)\right\rangle\right\rangle\right.$ is comeager in

$$
\left.\Sigma\left(\hat{s} g\left(n_{0}\right) \hat{\delta}\left(m_{0}\right) \hat{\cdots} \hat{g}\left(n_{k-1}\right) \hat{\delta}\left(m_{k-1}\right)\right)\right] \text {. }
$$

Define $\tilde{f}$ and $\tilde{g}$ as follows:

$$
\begin{gathered}
\tilde{f}(s)=\left\{\begin{array}{l}
\left\langle\alpha\left(n_{0}\right), \ldots, \alpha\left(n_{k-1}\right)\right\rangle \\
\text { arbitrary } \text { otherwise; }
\end{array} \text { if } s=\left\langle n_{0}, \ldots, n_{k-1}\right\rangle,\right. \\
\tilde{g}(s)=\left\{\begin{array}{l}
\beta\left(n_{0}\right) \hat{\beta}\left(n_{1}\right) \ldots \hat{\beta}\left(n_{k-1}\right) \\
\text { arbitrary } \text { otherwise. }
\end{array} \text { if } s=\left\langle n_{0}, \ldots, n_{k-1}\right\rangle,\right.
\end{gathered}
$$

The result now follows immediately.

ReMarK 5.4.1. The import of the Transfer Theorem is that if a set $E$ computed by $\Phi$ is such that $E^{\bullet \Sigma(s)}$ is computed by $\Psi$, then for any set $F$ computed by $\Phi^{*}, F^{* \Sigma(s)}$ is computed by $\Psi^{*}$

REMARK 5.4.2. It is clear from the proof of 5.4(c) that, under the hypothesis of the theorem, for any normal family $\left\{F_{p}\right\}$ of subsets of $\omega^{\omega} \times \omega^{\omega}$ with $F=R \Phi_{N}\left(\left\{F_{p}\right\}\right)$,

$$
F^{* \Sigma(s)}=R \Phi_{M}\left(\left\{F_{f^{\prime}(p)} \Sigma\left(\hat{s} g^{\prime}(p)\right): p \in \omega\right\}\right)
$$


where

$$
\begin{aligned}
& f^{\prime}\left(\left\langle n_{0}, \ldots, n_{k-1}\right\rangle\right)=\left\langle f\left(n_{0}\right), \ldots, f\left(n_{k-1}\right)\right\rangle, \\
& g^{\prime}\left(\left\langle n_{0}, \ldots, n_{k-1}\right\rangle\right)=g\left(n_{0}\right) \hat{g}\left(n_{1}\right) \hat{\jmath} \hat{g}\left(n_{k-1}\right) .
\end{aligned}
$$

Here, normality of $\Psi=\Phi_{M}$ is not required.

6. Applications. We shall now apply the Transfer Theorem to deduce Vaught's Formula for $E^{*}$ (cf. [19, Theorem 1.6]) and the Category Formula of Burgess [3].

TheOREM 6.1 (VAUght). Assume $E=\mathscr{A}\left(\left\{E_{n}: n \in \omega\right\}\right)$, with each $E_{n} \subset \omega^{\omega} \times \omega^{\omega}$ normal. Then $x \in E^{* \Sigma(s)}$ if and only if

$$
\begin{aligned}
&\left(\forall u_{0} \in \operatorname{Seq}\right)\left(\exists v_{0} \in \operatorname{Seq}\right)\left(\exists k_{0}\right)\left(\forall u_{1} \in \operatorname{Seq}\right)\left(\exists v_{1} \in \operatorname{Seq}\right)\left(\exists k_{1}\right) \cdots \\
&(\forall i)\left[E_{\left\langle k_{0}, \ldots, k_{1-1}\right\rangle}^{x} \text { is comeager in } \Sigma\left(\hat{s} \hat{u_{0}} \hat{v_{0}} \cdots \hat{u_{i-1} v_{i-1}}\right)\right] .
\end{aligned}
$$

Proof. Let $N=\{\{n\}: n \in \omega\}$ so that $\Phi_{N}=U$. Suppose $F=\Phi_{N}\left(\left\{F_{n}: n \in \omega\right\}\right)$ $=\cup_{n \in \omega} F_{n}$, where each $F_{n}$ is a normal subset of $\omega^{\omega} \times \omega^{\omega}$. Then,

$F^{x}$ is comeager in $\Sigma(s) \leftrightarrow(\forall u \in$ Seq $)\left[F^{x}\right.$ is nonmeager in $\left.\Sigma(\hat{s u})\right]$

$$
\begin{aligned}
& \leftrightarrow(\forall u \in \operatorname{Seq})(\exists k)\left[F_{k}^{x} \text { is nonmeager in } \Sigma(\hat{s u})\right] \\
& \leftrightarrow(\forall u \in \operatorname{Seq})(\exists k)(\exists v \in \operatorname{Seq})\left[F_{k}^{x} \text { is comeager in } \Sigma(\hat{s} \hat{u} v)\right] .
\end{aligned}
$$

Let $\Phi_{M}$ be a $\delta-s$ operation such that for any family $\left\{A_{n}\right\}$,

$$
\Phi_{M}\left(\left\{A_{n}: n \in \omega\right\}\right)=\bigcap_{u \in \text { Seq }} \bigcup_{u^{\prime} \in \text { Seq }} \bigcup_{k \in \omega} A_{\left\langle u,\left\langle k, v^{\prime}\right\rangle\right\rangle} .
$$

Hence,

$F^{x}$ is comeager in $\Sigma(s)$

$$
\begin{aligned}
& \leftrightarrow(\exists \eta \in M)(\forall n \in \eta)\left[F_{(n)_{1.0}^{x}}^{x} \text { is comeager in } \Sigma\left(\hat{s}(n)_{0}(n)_{1,1}\right)\right] \\
& \leftrightarrow(\exists \eta \in M)(\forall n \in \eta)\left[F_{f(n)}^{x} \text { is comeager in } \Sigma(\hat{s g}(n))\right],
\end{aligned}
$$

where $f(n)=(n)_{1,0}$ and $g(n)=(n)_{0}(n)_{1,1}$. Since

$$
E=\mathscr{A}\left(\left\{E_{n}: n \in \omega\right\}\right)=R \Phi_{N}\left(\left\{E_{n}\right\}\right),
$$

the Transfer Theorem immediately gives

$$
E^{* \Sigma(s)}=R \Phi_{M}\left(\left\{E_{\tilde{f}(p)}^{\Sigma(\hat{s} \tilde{g}(p))}: p \in \omega\right\}\right)
$$

where

$$
\tilde{g}\left(\left\langle n_{0}, \ldots, n_{k-1}\right\rangle\right)=g\left(n_{0}\right) \hat{g}\left(n_{1}\right) \hat{\cdots} \hat{g}\left(n_{k-1}\right)
$$

and $\tilde{f}\left(\left\langle n_{0}, \ldots, n_{k-1}\right\rangle\right)=\left\langle f\left(n_{0}\right), \ldots, f\left(n_{k-1}\right)\right\rangle$. Therefore,

$E^{x}$ is comeager in $\Sigma(s)$

$$
\begin{gathered}
\leftrightarrow\left(\exists \eta_{0} \in M\right)\left(\forall n_{0} \in \eta_{0}\right)\left(\exists \eta_{1} \in M\right)\left(\forall n_{1} \in \eta_{1}\right) \cdots \\
(\forall i)\left[E_{\left\langle f\left(n_{0}\right) \ldots,\left(n_{1-1}\right)\right\rangle}^{x} \text { is comeager in } \Sigma\left(\hat{s} g\left(n_{0}\right) \hat{\cdots} g\left(n_{i-1}\right)\right)\right] \\
\left(\forall u_{0} \in \operatorname{Seq}\right)\left(\exists v_{0} \in \operatorname{Seq}\right)\left(\exists k_{0}\right)\left(\forall u_{1} \in \operatorname{Seq}\right)\left(\exists v_{1} \in \operatorname{Seq}\right)\left(\exists k_{1}\right) \cdots \\
(\forall i)\left[E_{\left\langle k_{0} \ldots \ldots k_{-1}\right\rangle}^{x} \text { is comeager in } \Sigma\left(\hat{s} \hat{u_{0} v_{0}} \cdots \hat{u_{i-1}} v_{i-1}\right)\right] .
\end{gathered}
$$


DEFINITION 6.2. Define an operation $\mathscr{V}$ as follows.

$$
\begin{aligned}
& x \in \mathscr{V}\left(\left\{E_{n}: n \in \omega\right\}\right) \\
& \leftrightarrow\left(\forall u_{0} \in \operatorname{Seq}\right)\left(\exists v_{0} \in \operatorname{Seq}\right)\left(\exists k_{0}\right)\left(\forall u_{1} \in \operatorname{Seq}\right)\left(\exists v_{1} \in \operatorname{Seq}\right)\left(\exists k_{1}\right) \\
& \cdots(\forall i)\left[x \in E_{\left.\left\langle\left\langle u_{0} \cdot\left\langle k_{0}, v_{0}\right\rangle\right\rangle \ldots,\left\langle u_{t-1} \cdot\left\langle k_{1-1}, v_{1-1}\right\rangle\right\rangle\right\rangle\right] .}\right.
\end{aligned}
$$

Clearly $\mathscr{V}$ is positive analytical and $\mathscr{V} \sim \mathscr{A}$. Call $\mathscr{V}$ the Vaught operation.

Define a sequence of positive analytical operations $\left\{S_{\rho}: \rho<\omega_{1}\right\}$ by the induction

$$
S_{0}=\mathscr{V}, \quad S_{\rho+1}=S_{\rho}^{*} .
$$

If $\lambda$ is limit, choose $\rho_{i} \uparrow \lambda$ and set for any family $\left\{E_{n}\right\}$,

$$
\Psi\left(\left\{E_{n}\right\}\right)=\bigcap_{i \in \omega} \Phi_{M_{\rho_{1}} M_{\rho_{i}}^{0}}\left(\left\{E_{\langle i, m\rangle}: m \in \omega\right\}\right),
$$

where $M_{\rho_{1}}$ is the canonical base for $S_{\rho_{i}}$. Then define $S_{\lambda}=\Psi^{*}$. It is easy to check by induction that for each $\rho, R_{\rho} \sim S_{\rho}$ and hence $\mathscr{R}^{\rho}=\Sigma_{1}^{R_{\rho}}=\Sigma_{1}^{S_{\rho}}$.

We shall now deduce the Category Formula of Burgess by showing that if $E$ is in $\mathscr{R}^{\rho}$, then $E^{*}$ is computed by $S_{\rho}$.

THEOREM 6.3. Let $E \subseteq \omega^{\omega} \times \omega^{\omega}$ be a set in $\mathscr{R}^{\rho}$. Then $E^{\cdot \Sigma(s)}=\left\{x: E^{x}\right.$ is comeager in $\Sigma(s)$ ) is also in $\mathscr{R}^{p}$.

Proof. We shall prove the theorem by induction. Let $N_{\rho}$ and $M_{\rho}$ denote the canonical bases for $R_{\rho}$ and $S_{\rho}$, respectively.

Assume that for all $\nu<\rho$ there are functions $f_{\nu}$ and $g_{\nu}$ such that if

$$
F=R_{\nu}\left(\left\{F_{n}: n \in \omega\right\}\right),
$$

with each $F_{n} \subseteq \omega^{\omega} \times \omega^{\omega}$ normal, then

$$
F^{* \Sigma(s)}=S_{\nu}\left(\left\{F_{f_{\nu}(n)} \Sigma^{\left(\hat{s} g_{\nu}(n)\right)}: n \in \omega\right\}\right) .
$$

We shall then show that if $E=R_{\rho}\left(\left\{E_{n}\right\}\right)$, then $E^{* \Sigma(s)}$ is similarly computed by $S_{\rho}$. The result then follows by observing that for each clopen $E_{n}, E_{n}^{*}$ is also clopen.

Case 1. $\rho=\nu+1$. In this case, $E=R_{\nu}^{*}\left(\left\{E_{n}: n \in \omega\right\}\right)$. Hence by the Transfer Theorem,

$E^{x}$ is comeager in $\Sigma(s) \leftrightarrow\left(\exists \eta_{0} \in M_{\nu}\right)\left(\forall n_{0} \in \eta_{0}\right)\left(\forall \xi_{0} \in M_{0}\right)\left(\exists m_{0} \in \xi_{0}\right) \cdots$

$$
\begin{aligned}
& (\forall i)\left[E_{\tilde{f}_{\nu}\left(\left\langle\left\langle n_{0}, m_{0}\right\rangle \ldots,\left\langle n_{t-1}, m_{1-1}\right\rangle\right\rangle\right)}^{x}\right. \text { is comeager in } \\
& \left.\qquad\left(\hat{s} \tilde{g}_{\nu}\left(\left\langle\left\langle n_{0}, m_{0}\right\rangle, \ldots,\left\langle n_{i-1}, m_{i-1}\right\rangle\right\rangle\right)\right)\right]
\end{aligned}
$$

where $\tilde{f}_{\nu}$ and $\tilde{g}_{\nu}$ are related to $f_{\nu}$ and $g_{\nu}$ as in 5.4. Setting $f_{\rho}=\tilde{f}_{\nu}$ and $g_{\rho}=\tilde{g}_{\nu}$ we get

$$
E^{* \Sigma(s)}=S_{\rho}\left(\left\{E_{f_{\rho}(n)}^{* \Sigma\left(\hat{s}_{\rho}(n)\right)}: n \in \omega\right\}\right)
$$

Case 2. $\rho$ is limit. Choose a sequence $\rho_{i} \uparrow \rho$ and for any normal family $\left\{H_{n}: n \in \omega\right\}$ set

$$
H=\Phi\left(\left\{H_{n}\right\}\right)=\bigcap_{i \in \omega} \Phi_{N_{p_{i}} N_{p_{1}}^{0}}\left(\left\{H_{\langle i, n\rangle}: n \in \omega\right\}\right)
$$


Then,

$$
\begin{aligned}
H^{x} \text { is comeager in } \Sigma(s) \leftrightarrow & (\forall i)\left[\left(\Phi_{N_{\rho_{1} N_{\rho_{1}}^{0}}}\left(\left\{H_{\langle i, m\rangle}: m \in \omega\right\}\right)\right)^{x} \text { is comeager in } \Sigma(s)\right] \\
\leftrightarrow & (\forall i)\left(\exists \eta \in M_{\rho_{1}}\right)(\forall n \in \eta)\left(\forall \xi \in M_{\rho_{i}}\right)(\exists m \in \xi) \\
& {\left[H_{\left\langle i, \alpha_{1}(\langle n, m\rangle)\right\rangle}^{x} \text { is comeager in } \Sigma\left(\hat{s} \beta_{i}(\langle n, m\rangle)\right)\right] }
\end{aligned}
$$

(by the Transfer Theorem),

where $\alpha_{i}$ and $\beta_{i}$ are obtained as in 5.4(b). Therefore,

$$
H^{* \Sigma(s)}=\Psi\left(\left\{H_{f(m)}^{* \Sigma(\hat{s} g(m))}: m \in \omega\right\}\right) .
$$

where $\Psi$ is the operation in 6.2 and

$$
f(\langle i, n\rangle)=\left\langle i, \alpha_{i}(n)\right\rangle, \quad g(\langle i, n\rangle)=\beta_{i}(n) .
$$

Since $E=\Phi^{*}\left(\left\{E_{n}\right\}\right)$, by applying the Transfer Theorem again we obtain the result as in Case 1.

As the $R$-sets have the Baire property the next result follows immediately.

Corollary 6.4. If $E \in c \mathscr{R}^{\rho}$, then $E^{* \Sigma(s)}$ is also in $c \mathscr{R}^{\rho}$.

By repeatedly applying the Transfer Theorem and 6.3 to every level of the hierarchy of $\mathscr{B} \mathscr{R}^{\rho}$-sets one obtains

COROllaRy 6.5. If $E \in \mathscr{B} \mathscr{R}_{\mu}^{\rho}\left(\mu, \rho<\omega_{1}\right)$, then $E^{* \Sigma(s)}$ is also in $\mathscr{B} \mathscr{R}_{\mu}^{\rho}$. In particular, putting $\rho=0$, one has for any $C$-set $E, E^{*}$ is also a $C$-set.

\section{The approximation theorem.}

LEMMA 7.1. For $\rho<\omega_{1}$, let $R_{\rho}=\Phi_{K}=R \Phi_{N N^{0}}$. As in the proof of 6.3 there are functions $\tilde{f}$ and $\tilde{g}$ such that for any normal family $\left\{E_{n}\right\}$ with $E=R_{\rho}\left(\left\{E_{n}\right\}\right)$,

$$
\begin{aligned}
E^{x} \text { is comeager } \leftrightarrow & (\exists \eta \in \tilde{K})(\forall n \in \eta)\left[E_{\tilde{f}(n)}^{x} \text { is comeager in } \Sigma(\tilde{g}(n))\right] \\
\leftrightarrow & \left(\exists \eta_{0} \in M\right)\left(\forall n_{0} \in \eta_{0}\right) \cdots \\
& (\forall k)\left[E^{x}\left\langle\alpha(\langle n, m\rangle) \ldots, \alpha\left(\left\langle n_{k}, 1, m_{k-1}\right\rangle\right)\right\rangle\right. \text { is comeager in } \\
& \sum\left(\beta\left(\left\langle n_{0}, m_{0}\right\rangle\right) \hat{\cdots} \beta\left(\left\langle n_{k-1}, m_{k-1}\right\rangle\right)\right],
\end{aligned}
$$

where $\tilde{f}, \tilde{g}$ and $\alpha, \beta$ are related as in $5.4(\mathrm{c})$ and $\Phi_{M} \sim \Phi_{N}$ and $\tilde{K}=R M M^{0}$.

Then, one can choose $\tilde{K}$ such that for any $\eta \in \tilde{K}, \cup_{n \in \eta} \Sigma(\tilde{g}(n))$ is dense in $\omega^{\omega}$.

In fact, $\tilde{K}$ may be taken to be the canonical base for $S_{\rho}$ with the property that for any $\eta \in \tilde{K}$ and $s \in$ Seq there is an $n \in \eta$ such that $\tilde{g}(n)$ extends $s$.

Proof. We shall prove this by induction on $\rho$. For $\rho=0, \Phi_{\tilde{K}}$ is the Vaught operation $\mathscr{V}$. Taking $\tilde{K}$ to be the canonical base for $\mathscr{V}$ it is easy to check the assertion of the above theorem.

So assume $\rho>0$ and the assertion holds for all $\nu<\rho$.

Case 1. $\rho=\nu+1$. Then $\Phi_{N}=R_{\nu}$. Let $\eta \in \tilde{K}=R M M^{0}$, where $M$ has been chosen to satisfy the assertion of the theorem for the operation $R_{\nu}$. 
Fix a basic clopen set $\Sigma(t)$. We have to show that there is $n \in \eta$ such that $\tilde{g}(n)$ is consistent with $t$ (in fact, extends $t$ ).

Since $\eta \in R M M^{0}$,

$$
\begin{aligned}
\left(\exists \eta_{0} \in M\right)\left(\forall n_{0} \in \eta_{0}\right) & \left(\forall \xi_{0} \in M\right)\left(\exists m_{0} \in \xi_{0}\right) \cdots \\
& \cdots(\forall k)\left[\left\langle\left\langle n_{0}, m_{0}\right\rangle \cdots\left\langle n_{k-1}, m_{k-1}\right\rangle\right\rangle \in \eta\right] .
\end{aligned}
$$

Fix $\eta_{0} \in M$ such that for each $n_{0} \in \eta_{0}$,

$\left(\forall \xi_{0} \in M\right)\left(\exists m_{0} \in \xi_{0}\right)\left(\exists \eta_{1} \in M\right)\left(\forall n_{1} \in \eta_{1}\right)\left(\forall \xi_{1} \in M\right)\left(\exists m_{1} \in \xi_{1}\right) \cdots$

$$
(\forall k)\left[\left\langle\left\langle n_{0}, m_{0}\right\rangle, \ldots,\left\langle n_{k-1}, m_{k-1}\right\rangle\right\rangle \in \eta\right] .
$$

By the induction hypothesis, there is an $n_{0}^{*} \in \eta_{0}$ such that $g\left(n_{0}^{*}\right)$ extends $t$. Now pick a $\xi_{0} \in M$ such that for some $m_{0}^{*} \in \xi_{0}$,

$$
\begin{aligned}
\left(\exists \eta_{0} \in M\right)\left(\forall n_{0} \in \eta_{0}\right)\left(\forall \xi_{0} \in M\right)\left(\exists m_{0} \in \xi_{0}\right) \cdots \\
\cdots(\forall k)\left[\left\langle\left\langle n_{0}, m_{0}\right\rangle \cdots\left\langle n_{k-1}, m_{k-1}\right\rangle\right\rangle \in \eta\right] .
\end{aligned}
$$

Clearly, $n=\left\langle\left\langle n_{0}^{*}, m_{0}^{*}\right\rangle\right\rangle \in \eta$ and $\tilde{g}(n)=g\left(n_{0}^{*}\right) \hat{\delta}\left(m_{0}^{*}\right)$ (cf. 5.4). Thus $\tilde{g}(n)$ extends $t$.

Case 2. $\rho$ is limit. Let $\rho_{i} \uparrow \rho$. Then $R_{\rho}=\Phi_{N}^{*}=R \Phi_{N N^{0}}$, where $\Phi_{N}$ is the operation given by

$$
\Phi_{N}\left(\left\{F_{n}\right\}\right)=\bigcap_{i \in \omega} \Phi_{N_{p_{1}} N_{p_{i}}^{0}}\left(\left\{F_{\langle i, m\rangle}: m \in \omega\right\}\right) \text { and } \Phi_{N_{p_{1}}}=R_{\rho_{i}}
$$

In this case, if $E=\Phi_{N}\left(\left\{E_{n}\right\}\right)$, then

$$
\begin{aligned}
E^{x} \text { is comeager } \leftrightarrow & (\exists \eta \in \tilde{N})(\forall n \in \eta)\left[E_{\tilde{f}(n)}^{x} \text { is comeager in } \Sigma(\tilde{g}(n))\right] \\
\leftrightarrow & (\forall i)\left(\exists \eta \in M_{\rho_{1}}\right)(\forall n \in \eta)\left(\exists \xi \in M_{\rho_{1}}^{0}\right)(\forall m \in \xi) \\
& {\left[E_{\left\langle i, \alpha_{1}(\langle n, m\rangle)\right\rangle}^{x} \text { is comeager in } \Sigma\left(\beta_{i}(\langle n, m\rangle)\right)\right] }
\end{aligned}
$$

(cf. 6.3, Case 2).

In view of Case 1, it suffices to show that for any $\eta \in \tilde{N}$ and for any $\Sigma(t)$, there is an $n \in \eta$ such that $\tilde{g}(n)$ extends $t$.

Now, $\tilde{N}$ may be chosen such that

$$
\eta \in \tilde{N} \leftrightarrow(\forall i)\left(\exists \eta^{\prime} \in M_{\rho_{1}}\right)\left(\forall n \in \eta^{\prime}\right)\left(\exists \xi^{\prime} \in M_{\rho_{i}}^{0}\right)\left(\forall m \in \xi^{\prime}\right)[\langle i,\langle n, m\rangle\rangle \in \eta],
$$

where each $\boldsymbol{M}_{\rho_{1}}$ has been chosen to satisfy the assertion of the theorem.

Let $\eta \in \tilde{N}$ and fix any $i^{*} \in \omega$. Get $\eta^{*} \in M_{\rho_{i}}$ such that for each $n \in \eta^{*}$

$$
\left(\exists \xi^{\prime} \in M_{\rho_{i}}^{0}\right)\left(\forall m \in \xi^{\prime}\right)\left[\left\langle i^{*},\langle n, m\rangle\right\rangle \in \eta\right] .
$$

Since $\eta^{*} \in M_{\rho_{i},}$, by the induction hypothesis, there is an $n^{*} \in \eta^{*}$ such that $g_{i^{*}}\left(n^{*}\right)$ extends $t$. Get $\xi^{\prime} \in M_{\rho_{1}}^{0}$ and $m^{*} \in \xi^{\prime}$ such that $\left\langle i^{*},\left\langle n^{*}, m^{*}\right\rangle\right\rangle \in \eta$. Take $n=$ $\left\langle i^{*},\left\langle n^{*}, m^{*}\right\rangle\right\rangle$. Then

$$
\tilde{g}(n)=\beta_{i^{*}}\left(\left\langle n^{*}, m^{*}\right\rangle\right)=g_{i^{*}}\left(n^{*}\right)^{\hat{\delta}} \delta_{i^{*}}\left(m^{*}\right)
$$

(cf. 6.3) and hence $\tilde{g}(n)$ extends $t$. 
LEMMA 7.2. Let $\mathscr{F}$ be a $\sigma$-field closed under operation $\mathscr{A}$ and let $\mathscr{B}_{\omega^{\omega}}$ denote the Borel $\sigma$-field on $\omega^{\omega}$. Let $E \subseteq \omega^{\omega} \times \omega^{\omega}$. Suppose there is a family $\left\{A_{n}\right\}$ such that

(a) $A=\mathscr{A}\left(\left\{A_{n}\right\}\right)$,

(b) $A \subseteq E$,

(c) each $A_{n} \in \mathscr{F} \otimes \mathscr{B}_{\omega^{\omega}}$,

(d) $A^{x}$ is comeager whenever $E^{x}$ is comeager.

Then there is a set $B \in \mathscr{F} \otimes \mathscr{B}_{\omega^{\omega}}$ such that $B \subseteq E$ and $B^{x}$ is comeager whenever $E^{x}$ is comeager.

Proof. Let $I: \omega^{\omega} \rightarrow \omega^{\omega}$ be the characteristic function of a generator for a countably generated sub- $\sigma$-field $\mathscr{F}_{0}$ of $\mathscr{F}$ such that each $A_{n} \in \mathscr{F}_{0} \otimes \mathscr{B}_{\omega^{\omega}}$. Let $I\left(\omega^{\omega}\right)=$ $D$. Then, as is well known, $I$ is a bimeasurable function between $\mathscr{F}_{0}$ and $\mathscr{B}_{D}$, the Borel $\sigma$-field on $D$. Set

$$
\tilde{A}_{n}=\left\{(I(\alpha), \beta):(\alpha, \beta) \in A_{n}\right\}, \quad \tilde{A}=\{(I(\alpha), \beta):(\alpha, \beta) \in A\} .
$$

Clearly, $\tilde{A}_{n} \in \mathscr{B}_{D} \otimes \mathscr{B}_{\omega^{\omega}}$ and $A^{*}=\mathscr{A}\left(\left\{\tilde{A_{n}}\right\}\right)$. Hence $\tilde{A}$ is an analytic set in $D \times \omega^{\omega}$. Get an analytic set $C \subseteq \omega^{\omega} \times \omega^{\omega}$ such that

$$
\tilde{A}=C \cap\left(D \times \omega^{\omega}\right) \text {. }
$$

Then by 1.6 of [17], get $\tilde{B} \in \mathbb{Q} \otimes \mathscr{B}_{\omega^{\omega}}$, where $\mathbb{Q}$ is the analytic $\sigma$-field on $\omega^{\omega}$, such that $\tilde{B} \subseteq C$ and $\tilde{B}^{x}$ is comeager whenever $C^{x}$ is comeager. Put

$$
B=\{(\alpha, \beta):(I(\alpha), \beta) \in \tilde{B}\} .
$$

Since $\mathscr{F}$ is closed under operation $\mathscr{A}$, clearly $B \in \mathscr{F} \otimes \mathscr{B}_{\omega^{\omega}}$ and moreover, $B \subseteq E$ and $B^{x}$ is comeager whenever $E^{x}$ is comeager.

We have adapted the proof of the Category Formula [2] in the next lemma.

LEMMA 7.3. Suppose we have

$$
\begin{aligned}
\left(\forall s_{0}\right)\left(\exists t_{0}\right)\left(\forall s_{1}\right)\left(\exists t_{1}\right) \cdots & \left\{\left(\forall a_{0}\right)\left(\exists b_{0}\right)\left(\forall a_{1}\right)\left(\exists b_{1}\right) \cdots P(\alpha, \beta)\right\} \\
\leftrightarrow & \left(\forall s_{0}\right)\left(\forall a_{0}\right)\left(\exists t_{0}\right)\left(\exists b_{0}\right)\left(\forall s_{1}\right)\left(\forall a_{1}\right)\left(\exists t_{1}\right)\left(\exists b_{1}\right) \cdots P(\alpha, \beta),
\end{aligned}
$$

where $\alpha=\left(a_{0}, b_{0}, a_{1}, b_{1}, \ldots\right), a_{i}, b_{i} \in \omega$, and $\beta=\hat{s_{0} t_{0}} \hat{s_{1} t_{1}} \cdots ; s_{i}, t_{i} \in$ Seq.

If $\exists$ wins the second game, then he may do so by means of a strategy $\sigma^{*}$ such that, to every complete play $s_{0}, a_{0}, t_{0}, b_{0}, \ldots$ consistent with $\sigma^{*}$, there corresponds a complete play $s_{0}^{\prime}, t_{0}^{\prime}, s_{1}^{\prime} . t_{1}^{\prime} \ldots$ consistent with a winning strategy for $\exists$ in the first game such that

$$
\hat{s_{0}} \hat{t_{0}} \hat{s_{1}} \hat{t_{1}} \cdots=s_{0}^{\prime \wedge} t_{0}^{\prime} \hat{s_{1}^{\prime}} t_{0}^{\prime \wedge} \cdots \text {. }
$$

Proof. Let $w_{-1}, w_{0}, w_{1}, w_{2}, \ldots$ be an enumeration of $\omega^{<\omega}$ such that $w_{-1}$ is the empty sequence and if $w_{i}$ is an initial segment of $w_{j}$, then $i<j$. For $s \in \omega^{<\omega}$, its code, denoted by $|s|$, is its position in the enumeration.

Suppose $\exists$ wins the second game with strategy $\sigma$. We shall now construct a winning strategy $\tau$ for player II in the first (Banach-Mazur) game. $\tau$ will be defined (by induction) in such a way that every partial play consistent with $\tau$ corresponds to a partial play consistent with $\boldsymbol{\sigma}$.

Suppose $s_{0}, t_{0}, \ldots, s_{n-1}, t_{n-1}$ have been defined consistent with $\tau$ and

$$
\tau\left(s_{0}, t_{0}, \ldots, s_{n-1}, t_{n-1}, s\right)
$$


has not been defined. Let $n$ be the code of $\left(a_{0}, \ldots, a_{m-1}, a\right)$ and let $\left|\left(a_{0}, \ldots, a_{m-1}\right)\right|$ $=n^{*}$. Clearly, $n^{*}<n$. Let the partial play (consistent with $\sigma$ ) corresponding to $\left(s_{0}, t_{0}, \ldots, s_{n^{*}}, t_{n^{*}}\right)$ be $\left(a_{0}, u_{0}, \ldots, a_{m-1}, u_{m-1}, b_{m-1}, v_{m-1}\right)$ such that $\hat{s_{0}} \hat{t_{0}} \cdots \hat{s_{n^{*}}} t_{n^{*}}$ $=\hat{u_{0} v_{0}} \cdots \hat{u_{m-1}} v_{m-1}$. Put

$$
u=\hat{s_{n^{*}+1}} \hat{t_{n^{*}+1}} \cdots \hat{s_{n-1}} \hat{t_{n-1}} s .
$$

Let

$$
\sigma\left(a_{0}, u_{0} ; b_{0}, v_{0} ; \cdots ; b_{m-1}, v_{m-1}, a, u\right)=(b, v)
$$

Then

$$
\tau\left(s_{0}, t_{0}, \ldots, s_{n-1}, t_{n-1}, s\right)=v,
$$

and the partial play associated with

$$
s_{0}, t_{0}, \ldots, s_{n-1}, t_{n-1}, s, v
$$

is

$$
a_{0}, u_{0} ; b_{0}, v_{0}, \ldots, b_{m-1}, v_{m-1} ; a, u ; b, v .
$$

We shall now show that $\tau$ is a winning strategy for player II in the Banach-Mazur game. Let

$$
s_{0}, t_{0}, s_{1}, t_{1}, s_{2}, t_{2}, \ldots
$$

be a complete play consistent with $\tau$. We shall have to show that

$$
\left(\forall a_{0}\right)\left(\exists b_{0}\right)\left(\forall a_{1}\right)\left(\exists b_{1}\right) \cdots P(\alpha, \beta),
$$

where

$$
\alpha=\left(a_{0}, b_{0}, a_{1}, b_{1}, \ldots\right) \text { and } \beta=\hat{s_{0} t_{0}} \hat{s_{1}} \hat{t_{1}} \cdots \text {. }
$$

So let $\forall$ play $a_{0}$. Suppose $\left|\left(a_{0}\right)\right|=n_{0}$. By definition of $\tau$, the partial play (consistent with $\sigma$ ) corresponding to $s_{0}, t_{0}, \ldots, s_{n_{0}}, t_{n_{0}}$ is

$$
a, \hat{s_{0} \hat{t}_{0}} \cdots \hat{s}_{n_{0}} ; b_{0}, t_{n_{0}}
$$

for some $b_{0} \in \omega . \exists$ replies with $b_{0}$. Next suppose $\forall$ plays $a_{1}$ and let $\left|\left(a_{0}, a_{1}\right)\right|=n_{1}$. By definition of $\tau$, the partial play (consistent with $\sigma$ ) corresponding to

$$
s_{0}, t_{0}, \ldots, s_{n_{0}}, t_{n_{0}}, \ldots, s_{n_{1}}, t_{n_{1}}
$$

is

$$
a_{0}, \underbrace{\hat{s_{0}} \hat{t_{0}} \cdots \hat{s}_{n_{0}}}_{u_{0}} ; b_{0}, t_{n_{0}}=v_{0} ; a_{1}, \underbrace{\hat{s_{n_{0}+1}} \hat{t_{n_{0}+1}} \cdots \hat{s}_{n_{1}}}_{u_{1}} ; b_{1}, t_{n_{1}}=v_{1} .
$$

$\exists$ replies with $b_{1}$ and the play proceeds as described. Since the play $a_{0}, \nu_{0} ; b_{0}, v_{0}$; $a_{1}, u_{1} ; b_{1}, v_{1} ; \ldots$ is consistent with $\sigma$, we have

$$
P\left(\alpha, \hat{u_{0}} \hat{v_{0}} \hat{u_{1}} \hat{v_{1}} \cdots\right) \text {. }
$$

But $\beta=\hat{u_{0}} \hat{v_{0}} \hat{u_{1}} \hat{v_{1}} \cdots$. Hence $P(\alpha, \beta)$. Consequently, $\tau$ is a winning strategy for II in the Banach-Mazur game.

We shall now modify $\sigma$ to $\sigma^{*}$ such that any complete play consistent with $\sigma^{*}$ corresponds to a complete play consistent with $\tau$. 
Definition of $\sigma^{*}$. Let $\forall$ play $a_{0}, s_{0}^{0}$ and suppose $\left|\left(a_{0}\right)\right|=n_{0}$. Simulate the following partial play in the Banach-Mazur game, where II plays with strategy $\tau, e^{*}$ being a fixed sequence number:

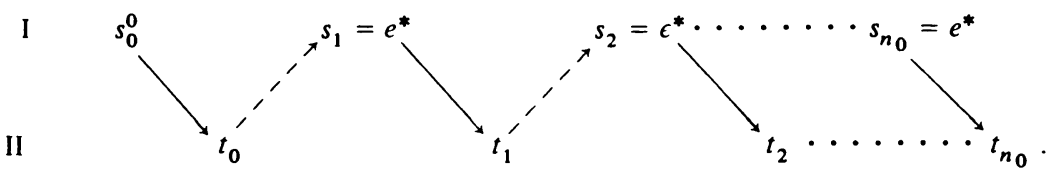

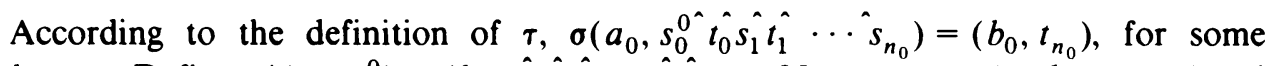
$b_{0} \in \omega$. Define $\sigma^{*}\left(a_{0}, s_{0}^{0}\right)=\left(b_{0}, \hat{t_{0}} \hat{s_{1}} \hat{t_{1}} \cdots \hat{s_{n_{0}}} t_{n_{0}}\right)$. Next suppose $\forall$ plays $a_{1}, s_{1}^{\prime}$ and let $\left|\left(a_{0}, a_{1}\right)\right|=n_{1}$. Consider the following partial play consistent with $\tau$ :

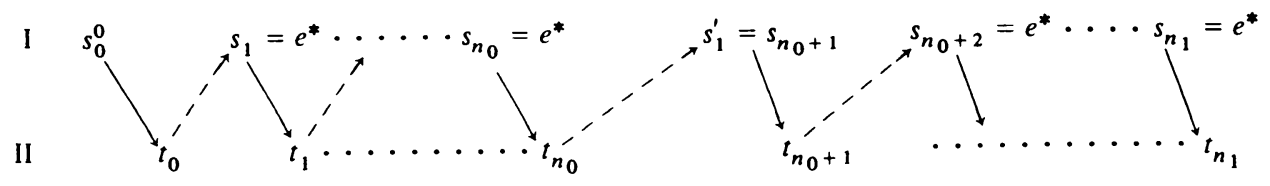

By definition of $\tau, \sigma\left(a_{0}, s_{0}^{0 \wedge} \hat{t_{0}} \cdots \hat{s_{n_{0}}} ; b_{0}, t_{n_{0}} ; a_{1}, \hat{s_{n_{0}+1}} \hat{t_{n_{0}+1}} \cdots \hat{s_{n_{1}}}\right)=\left(b_{1}, t_{n_{1}}\right)$, $b_{1} \in \omega$. Define

$$
\sigma^{*}\left(a_{0}, s_{0}^{0} ; \hat{b_{0}}, \hat{t_{0}} \hat{s_{1}} \cdots \hat{s_{n_{0}} t_{n_{0}}} ; a_{1}, s_{1}^{\prime}\right)=\left(\hat{b_{1}}, \hat{t_{n_{0}}+1} \hat{s_{n_{0}}+2} \cdots \hat{s_{n_{1}}} t_{n_{1}}\right)
$$

and so on. We shall show that $\sigma^{*}$ is a winning strategy for $\exists$ in the second game. First observe that any complete play

$$
a_{0}, u_{0}, b_{0}, v_{0}, a_{1}, u_{1}, b_{1}, v_{1}, \ldots
$$

consistent with $\sigma^{*}$ corresponds to a complete play

$$
s_{0}, t_{0}, s_{1}, t_{1}, \ldots
$$

consistent with $\tau$ such that

$$
\hat{s_{0}} \hat{t_{0}} \hat{s_{1}} \hat{t_{1}} \cdots=\hat{u_{0}} \hat{v_{0}} \hat{u_{1}} \hat{v_{1}} \cdots=\beta,
$$

say. To prove that $\sigma^{*}$ is a winning strategy, we have to show $P(\alpha, \beta)$, where $\alpha=\left(a_{0}, b_{0}, a_{1}, b_{1}, \ldots\right)$. Next observe that any play consistent with $\sigma^{*}$ is of the form

$$
\text { ( } \forall) \mathrm{I} \quad a_{0}, s_{0}^{0}
$$

$$
a_{1}, s_{1}^{\prime}=s_{n_{0}+1} \quad \cdots
$$

$$
b_{0}, \underbrace{\hat{t_{0}} \hat{s_{1}} \hat{t_{1}} \cdots \hat{s_{n_{0}}} t_{n_{0}}} \quad b_{1}, \underbrace{\hat{t_{n_{0}+1}} \cdots \hat{s_{n_{1}}} t_{n_{1}}} \cdots
$$

such that

$$
\begin{aligned}
& \text { ( } \forall) \mathrm{I} \quad a_{0}, s_{0}^{0 \wedge} \hat{t_{0}} \cdots \hat{s_{n_{0}}} \quad a_{1}, \hat{s_{n_{0}+1}} \hat{t_{n_{0}+1}} \cdots \hat{s_{n_{1}}} \\
& \text { (ヨ)II } \\
& b_{0}, t_{n_{0}} \\
& b_{1}, t_{n_{1}} \cdots
\end{aligned}
$$

is consistent with $\sigma$. Consequently, we have

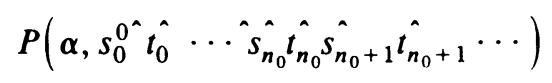

and hence $P(\alpha, \beta)$. 
REMARK 7.3.1. The definition of $\sigma^{*}$ is highly constructive and can be effected by a Borel function from the space of strategies to the space of strategies i.e., the map $\sigma \mapsto \sigma^{*}$ is Borel.

Using the above result, one immediately has the following.

COROLlaRY 7.4. If the second game in 7.3 is determined, then

$$
\begin{aligned}
\left(\exists s_{0}\right)\left(\forall t_{0}\right)\left(\exists s_{1}\right) & \left(\forall t_{1}\right) \cdots\left\{\left(\exists a_{0}\right)\left(\forall b_{0}\right)\left(\exists a_{1}\right)\left(\forall b_{1}\right) \cdots \neg P(\alpha, \beta)\right\} \\
\leftrightarrow & \left(\exists s_{0}\right)\left(\exists a_{0}\right)\left(\forall t_{0}\right)\left(\forall b_{0}\right)\left(\exists s_{1}\right)\left(\exists a_{1}\right)\left(\forall t_{1}\right)\left(\forall b_{1}\right) \cdots \neg P(\alpha, \beta),
\end{aligned}
$$

and the conclusion of 7.3 also holds here.

REMARK. Lemma 7.3 and Corollary 7.4 yield a constructive proof of a particular case of the Game Formula of Kechris [11].

LEMMA 7.5. Suppose $E \subseteq \omega^{\omega}$ be a set in $\mathscr{R}^{\rho}, \rho<\omega_{1}$ and let $R_{\rho}=R \Phi_{N}$. Assume $E=R_{p}\left(\left\{E_{n}\right\}\right)$, where each $E_{n}$ is clopen. Then,

$E$ is comeager $\leftrightarrow\left(\exists \eta_{0} \in M\right)\left(\forall n_{0} \in \eta_{0}\right)\left(\exists \eta_{1} \in M\right)\left(\forall n_{1} \in \eta_{1}\right) \cdots$

$$
(\forall k)\left[E_{\left\langle f\left(n_{0}\right), \ldots, f\left(n_{k-1}\right)\right\rangle} \text { is comeager in } \Sigma\left(g\left(n_{0}\right) \hat{g}\left(n_{1}\right) \hat{\cdots} \dot{g}\left(n_{k-1}\right)\right)\right]
$$

where $f$ and $g$ are some suitable functions and $\Phi_{M} \sim \Phi_{N}$. Moreover, $f$ and $g$ can be chosen such that, with any winning strategy o for $\exists$ one can associate a winning strategy $\sigma^{*}$ such that for any run $\eta_{0}, n_{0}, \eta_{1}, n_{1}, \ldots$ consistent with $\sigma^{*}$, the sequence $\beta=$ $g\left(n_{0}\right) \hat{g}\left(n_{1}\right) \hat{n}$ is in $E$.

Proof. The first assertion follows immediately from the proof of Theorem 6.3. Moreover, by Lemma 7.1, $M$ may be chosen such that for any $\eta \in M$ and $s$ there is $n \in \eta$ such that $g(n)$ extends $s$. Therefore, we have

$$
\begin{aligned}
& \left(\exists \eta_{0} \in M\right)\left(\forall n_{0} \in \eta_{0}\right) \cdots(\forall k)\left[E_{\left\langle f\left(n_{0}\right), \ldots f\left(n_{k-1}\right)\right\rangle}\right. \text { is comeager in } \\
& \left.\qquad \Sigma\left(g\left(n_{0}\right) \hat{g}\left(n_{1}\right) \hat{\cdots} \dot{g}\left(n_{k-1}\right)\right)\right] \\
& \leftrightarrow\left(\exists \eta_{0} \in M\right)\left(\forall n_{0} \in \eta_{0}\right) \cdots(\forall k)\left[\beta \in E_{\left\langle f\left(n_{0}\right) \ldots, f\left(n_{k-1}\right)\right\rangle}\right]
\end{aligned}
$$

where $\beta=g\left(n_{0}\right) \hat{g}\left(n_{1}\right) \hat{\ldots}$. Hence,

$$
\begin{aligned}
E \text { is comeager } \leftrightarrow\left(\exists \eta_{0} \in M\right)\left(\forall n_{0} \in \eta_{0}\right)\left(\exists \eta_{1} \in M\right)\left(\forall n_{1} \in \eta_{1}\right) \ldots \\
(\forall k)\left[g\left(n_{0}\right) \hat{g}\left(n_{1}\right) \hat{n} \in E_{\left\langle f\left(n_{0}\right) \ldots, f\left(n_{k-1}\right)\right\rangle}\right] .
\end{aligned}
$$

We shall prove the second assertion only for $\rho=1$, since for higher levels the proof involves similar ideas, although notationally cumbersome.

First observe that if $F=\mathscr{A}\left(\left\{F_{n}\right\}\right)$, with each $F_{n}$ clopen, then by the Category Formula [2], we have

$$
\begin{aligned}
& F \text { is meager } \leftrightarrow\left(\forall k_{0}\right)\left(\forall s_{0}\right)\left(\exists t_{0}\right)\left(\forall k_{1}\right)\left(\forall s_{1}\right)\left(\exists t_{1}\right) \ldots
\end{aligned}
$$

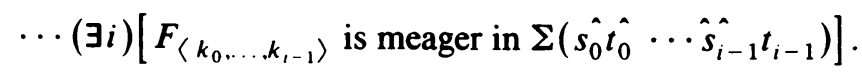


Therefore, whenever $G=\Gamma\left(\left\{G_{n}\right\}\right)$, where $\Gamma=\mathscr{A}^{0}$,

$G$ is comeager $\leftrightarrow\left(\forall k_{0}\right)\left(\forall s_{0}\right)\left(\exists t_{0}\right)\left(\forall k_{1}\right)\left(\forall s_{1}\right)\left(\exists t_{1}\right) \cdots$

$(\exists i)\left[G_{\left\langle k_{0} \ldots k_{t-1}\right\rangle}\right.$ is comeager in $\left.\Sigma\left(\hat{s_{0} \hat{t}_{0} \cdots \hat{s_{i-1}} t_{i-1}}\right)\right]$.

Since $E=R \Gamma\left(\left\{E_{n}\right\}\right)$, by 5.4 .2 we have

$$
\begin{aligned}
& E \text { is comeager } \leftrightarrow\left(\forall k_{0}^{0}\right)\left(\forall s_{0}^{0}\right)\left(\exists t_{0}^{0}\right)\left(\forall k_{1}^{0}\right)\left(\forall s_{1}^{0}\right)\left(\exists t_{1}^{0}\right) \cdots\left(\exists i_{0}\right) \\
& \left(\forall k_{0}^{1}\right)\left(\forall s_{0}^{1}\right)\left(\exists t_{0}^{1}\right)\left(\forall k_{1}^{1}\right)\left(\forall s_{1}^{1}\right)\left(\exists t_{1}^{1}\right) \cdots\left(\exists i_{1}\right) \\
& \left(\forall k_{0}^{2}\right)\left(\forall s_{0}^{2}\right)\left(\exists t_{0}^{2}\right)\left(\forall k_{1}^{2}\right)\left(\forall s_{1}^{2}\right)\left(\exists t_{1}^{2}\right) \cdots\left(\exists i_{2}\right) \cdots(\forall j) \\
& {\left[E_{\left\langle\left\langle k_{0}^{0}, k_{1}^{0} \ldots, k_{i^{-1}}^{0}\right\rangle \ldots,\left\langle k_{0}^{\prime-1} \ldots \ldots k_{t_{-1}}^{\prime-1}-1\right\rangle\right\rangle}\right. \text { is comeager in }} \\
& \left.\Sigma\left(s_{0}^{0^{\wedge}} t_{0}^{0^{\wedge}} \cdots \hat{s}_{i_{0}-1}^{0^{\wedge}} t_{i_{0}-1}^{0^{\wedge}} \cdots \hat{s} s_{0}^{-1^{\wedge}} t_{0}^{j-1} \cdots \hat{s}_{i_{-1}-1}^{j-1} t_{i_{j-1}-1}^{j-1}\right)\right] .
\end{aligned}
$$

Now, $E$ is comeager $\leftrightarrow$ II wins the game $G^{* *}\left(E^{c}\right) \leftrightarrow\left(\forall s_{0}\right)\left(\exists t_{0}\right)\left(\forall s_{1}\right)\left(\exists t_{1}\right) \cdots$ $[\beta \in E]$, where $\beta=\hat{s_{0}} \hat{t_{0}} \hat{s_{1}} \hat{t_{1}} \ldots$ Hence (using (i)), the equivalence in (ii) reduces to

(iii) $\left(\forall s_{0}\right)\left(\exists t_{0}\right)\left(\forall s_{1}\right)\left(\exists t_{1}\right) \cdots\left\{\left(\forall \alpha_{0}\right)\left(\exists n_{0}\right)\left(\forall \alpha_{1}\right)\left(\exists n_{1}\right) \cdots\right.$

$$
\begin{aligned}
& \left.(\forall j)\left[\beta \in E_{\left\langle\bar{\alpha}_{0}\left(n_{0}\right) \ldots \ldots \bar{\alpha}_{j-1}\left(n_{j-1}\right)\right\rangle}\right]\right\} \\
& \leftrightarrow\left(\forall k_{0}^{0}\right)\left(\forall s_{0}^{0}\right)\left(\exists t_{0}^{0}\right)\left(\forall k_{1}^{0}\right)\left(\forall s_{1}^{0}\right)\left(\exists t_{1}^{0}\right) \cdots\left(\exists i_{0}\right) \\
& \left(\forall k_{0}^{1}\right)\left(\forall s_{0}^{1}\right)\left(\exists t_{0}^{1}\right)\left(\forall k_{1}^{1}\right)\left(\forall s_{1}^{1}\right)\left(\exists t_{1}^{1}\right) \cdots\left(\exists i_{1}\right) \cdots(\forall j) \\
& {\left[s_{0}^{0 \wedge} t_{0}^{0} \cdots \hat{s} s_{i_{0}-1}^{0^{\wedge}} t_{i_{0}-1}^{0^{\wedge}} s_{0}^{1 \wedge} t_{0}^{1} \cdots \hat{s_{i_{1}-1}^{1} t_{i_{1}-1}^{1} \cdots}\right.} \\
& \left.\in E_{\left\langle\left\langle k_{0} \ldots \ldots k_{10}^{0}, 1\right\rangle \ldots\left\langle k_{0}^{\prime-1} \ldots, k_{1,-1}^{\prime-1}-1\right\rangle\right\rangle}\right] \text {. }
\end{aligned}
$$

As in $[3, \S 11]$, we shall define a game $G^{\prime}$ of length $\omega$ such that $\exists$ wins $G^{\prime}$ iff $\exists$ wins the second game in (iii). The game $G^{\prime}$ is as follows. Though its total length is $\omega$ we think of it as consisting of potentially infinite sequence of subgames each consisting of potentially infinite sequence of rounds. If in any play of $G^{\prime}$ the $j$ th subgame actually goes through infinitely many rounds, then the $(j+1)$ th subgame never gets started. This keeps the total length within bounds.

In the $j$ th subgame the two players play the rounds of that subgame. The $l$ th such round opens with $\exists$ signalling (by a choice of 0 or 1 ) either a challenge or a pass. If he challenges, the whole $j$ th subgame ends at once and the players proceed to the $(j+1)$ th subgame; in this case we record $u_{j}=\left\langle k_{0}^{j}, \ldots, k_{l-1}^{j}\right\rangle$ and

$$
v_{j}=s_{0}^{j} t_{0}^{j} \cdots \hat{s} \hat{l}_{-1}^{\hat{\imath}} t \hat{l}_{-1}^{j}
$$

formed from the moves. If $\exists$ passes, $\forall$ chooses $k j \in \omega$ and a sequence number $\left.s_{j}\right\}$ and $\exists$ replies with $t_{j}^{j} \in$ Seq; then the players proceed to the $(l+1)$ th round.

If some $j$ th subgame goes on forever because $\exists$ fails to challenge on any round, $\exists$ forfeits the game. If this provision does not apply, then a sequence $\left(u_{0}, v_{0}\right.$; $u_{1}, v_{1}, \ldots$ ) will have been generated. $\exists$ wins iff for all $j$

$$
\hat{v_{0} \hat{v_{1}} \hat{v_{2}}} \hat{\cdots}=\beta \in E_{\left\langle u_{0}, u_{1}, \ldots, u_{1}\right\rangle} \text {. }
$$


Thus the game $G^{\prime}$ is essentially of the form

$$
\left(\forall s_{0}\right)\left(\forall a_{0}\right)\left(\exists t_{0}\right)\left(\exists b_{0}\right)\left(\forall s_{1}\right)\left(\forall a_{1}\right)\left(\exists t_{1}\right)\left(\exists b_{1}\right) \cdots M(\alpha, \beta),
$$

where the condition $M$ is Borel (in fact $G_{\delta}$, cf. $[3, \S 11]$ ). We shall now make a few observations. First, observe that each subgame in the second game of (iii) is a Banach-Mazur game interlaced with operation $\Gamma$ (which may be looked upon as a game!). Secondly, the condition within [ ] is the same as the condition in the first game. Consequently, any reduction of the second game of (iii) involves the interlacing of a Banach-Mazur game with an ordinary game of length $\omega$ which is obtained by the corresponding reduction of the operation $R_{1}$. Therefore, reducing the $R_{1}$ operation within \{\} in the first game of (iii) as above, we observe that the equivalence (iii) is equivalent to the following:

$$
\begin{aligned}
\left(\forall s_{0}\right)\left(\exists t_{0}\right)\left(\forall s_{1}\right)\left(\exists t_{1}\right) \cdots\left\{\left(\forall a_{0}\right)\left(\exists b_{0}\right) \cdots M(\alpha, \beta)\right\} & \cdots\left(\forall s_{0}\right)\left(\forall a_{0}\right)\left(\exists t_{0}\right)\left(\exists b_{0}\right) \cdots M(\alpha, \beta) .
\end{aligned}
$$

Now, if $\exists$ wins the second game in (iii), he also wins $G^{\prime}$, the second game in (iv). By Lemma 7.3, $\exists$ wins with a strategy $\sigma$ such that for any complete play $s_{0}, a_{0}, t_{0}, b_{0}, \ldots$ consistent with $\sigma, \beta=\hat{s_{0}} \hat{t_{0}} \cdots$ corresponds to a complete play of the Banach-Mazur game in (iv). Moreover, the strategy $\sigma$ gives rise to a strategy $\sigma^{*}$ in the second game of (iii) such that, if $s_{0}^{0}, t_{0}^{0}, k_{0}^{0}, \ldots, i_{0} ; s_{0}^{1}, t_{0}^{1}, k_{0}^{1}, \ldots, i_{1} ; \cdots$ is a complete run consistent with $\sigma^{*}$, then the sequence $\beta=s_{0}^{0^{\wedge}} t_{0}^{0^{\wedge}} \cdots \hat{s} s_{i_{0}-1}^{0^{\wedge}} t_{i_{0}}^{0^{\wedge}} s_{0}^{1^{\wedge}} t_{0}^{1} \cdots$ is also produced by a complete run of the game $G^{\prime}$, when $\exists$ plays with $\sigma$. Consequently, $\beta$ satisfies the condition within \{\} in (iv) and as observed above, $\beta \in E$.

Remark. For sets $E$ at the higher level we have an equivalence similar to (iii). On the left side of the equivalence we have a Banach-Mazur game followed by the corresponding $R$-operation, and on the right side we have Banach-Mazur games "interlaced" with the operation (regarded as a game played on $\omega$ ) with dummy moves, if necessary. As in the proof above, the second game can be reduced to a game of length $\omega$ (see [3]) and the proof proceeds exactly as above.

REMARKS 7.6 (ON THE $\Delta$-TRANSFORM). We shall now look at the dual of the *-transform and obtain some analogous results. The proofs being very similar to what we have already done, we shall omit the details.

For a set $E \subseteq X \times Y$ and $U \subseteq Y$, put $E^{\Delta U}=\left\{x \in X: E^{x}\right.$ is nonmeager in $\left.U\right\}$.

Note that $\Delta$ is the dual of the *-transform in the sense that $E^{\Delta U}=\left[\left(E^{c}\right)^{* U}\right]^{c}$. The $\Delta$-transform behaves very much like the *-transform and we have the counterpart of Lemma 5.3:

With notation as in Lemma 5.3, we have

$$
E^{\Delta \Sigma(s)}=\bigcap_{\mu<\omega_{1}}\left[E_{e}^{\mu}\right]^{\Delta \Sigma(s)}=\bigcup_{\mu<\omega_{1}}\left[E_{e}^{\mu}-T^{\mu}\right]^{\Delta \Sigma(s)}
$$

This decomposition of the set $E^{\Delta}$ suggests, as in the case of $E^{*}$, that $\left(E^{\Delta}\right)^{c}$ may be obtained as a fixed point of an inductive operator. Indeed we have the following counterpart of Theorem 5.4. 
THEOREM. Let $\Phi_{N}$ and $\Phi_{M}$ be two $\delta$ - s operations such that $\Phi_{N}$ preserves the Baire property and $\Phi_{M}$ is normal and subsumes both (countable) $\cup$ and $\cap$. Suppose there are functions $f_{1}$ and $g_{1}$ such that for any normal family $\left\{E_{p}\right\}$ of subsets of $\omega^{\omega} \times \omega^{\omega}$ with $E=\Phi_{N}\left(\left\{E_{p}\right\}\right)$

$$
E^{\Delta \Sigma(s)}=\Phi_{M}\left(\left\{E_{f_{1}(p)}^{\Delta \Sigma\left(\hat{s} g_{1}(p)\right)}: p \in \omega\right\}\right) .
$$

Then for any regular normal family $\left\{F_{p}\right\}$, if $F=R \Phi_{N}\left(\left\{F_{p}\right\}\right)$ then $F^{x}$ is nonmeager in $\Sigma(s)$

$$
\begin{aligned}
& \leftrightarrow\left(\exists \eta_{0} \in M^{\prime}\right)\left(\forall n_{0} \in \eta_{0}\right)\left(\exists \eta_{1} \in M^{\prime}\right)\left(\forall n_{1} \in \eta_{1}\right) \cdots
\end{aligned}
$$

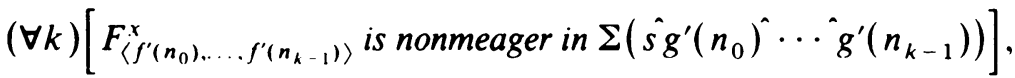

where $f^{\prime}$ and $g^{\prime}$ are suitable functions (independent of $\left\{F_{p}\right\}$ ) and $\Phi_{M^{\prime}}=\Phi_{M} \cup \cap$.

Proof. First observe that for any regular family $\left\{F_{p}\right\}, F_{s}^{\mu} \subseteq F_{t}$ if $t \prec s$. As in Theorem 5.4(c) we define a set relation operative on $\omega$ as follows:

$t \in \Gamma_{x}(A) \leftrightarrow F_{(t)_{0}}^{x}$ is meager in $\Sigma\left((t)_{1}\right) \vee$

$$
(\forall \eta \in M)(\exists n \in \eta)(\forall u)(\exists v)\left[\left\langle(t)_{0}\left\langle f_{1}(n)\right\rangle,(t) \hat{i} g_{1}(n) \hat{u} v\right\rangle \in A\right] .
$$

To obtain the result, one then shows that $x \in F^{\Delta \Sigma(s)} \leftrightarrow\langle e, s\rangle \notin \Gamma_{x}^{\infty}$. As in 6.3, this theorem immediately implies that for a set $E \in \mathscr{R}^{\rho}$, if $E=R \Phi_{N}\left(\left\{E_{n}\right\}\right)$, then

$$
\begin{aligned}
x \in E^{\Delta} \leftrightarrow\left(\exists \eta_{0} \in M\right)\left(\forall n_{0} \in \eta_{0}\right)\left(\exists \eta_{1} \in M\right)\left(\forall n_{1} \in \eta_{1}\right) \cdots \\
(\forall k)\left[E _ { \langle f ^ { \prime } ( n _ { 0 } ) \ldots f ^ { \prime } ( n _ { k - 1 } ) \rangle } ^ { x } \text { is nonmeager in } \Sigma \left(g^{\prime}\left(n_{0}\right) \cdots \hat{\left.\left.g^{\prime}\left(n_{k-1}\right)\right)\right],}\right.\right.
\end{aligned}
$$

for suitable $M, f^{\prime}, g^{\prime}$. Moreover, one can choose $M$ such that

$$
\eta \in M^{0} \& s \in \text { Seq } \rightarrow(\exists n \in \eta)\left(g^{\prime}(n) \text { extends } s\right) .
$$

Finally, observe that by (9) we have for $E \subseteq \omega^{\omega}$

(v) $E$ is meager $\leftrightarrow\left(\exists \eta_{0} \in M^{0}\right)\left(\forall n_{0} \in \eta_{0}\right)\left(\exists \eta_{1} \in M^{0}\right)\left(\forall n_{1} \in \eta_{1}\right) \cdots$

$$
\left.\left.(\exists k)\left[g^{\prime}\left(n_{0}\right) \hat{g^{\prime}}\left(n_{1}\right) \hat{\cdots} \notin E_{\left\langlef ^ { \prime } ( n _ { 0 } ) \ldots f ^ { \prime } \left( n_{k}\right.\right.},\right)\right\rangle\right] \text {. }
$$

Arguing as in Lemma 7.5 and invoking Corollary 7.4, we may show that $\exists$ can win the game (v) (if he does so) by a strategy $\sigma^{* *}$ such that if $\eta_{0}, n_{0}, \eta_{1}, n_{1}, \ldots$ is a complete play consistent with $\sigma^{* *}$, then $\beta=g^{\prime}\left(n_{0}\right) g^{\prime}\left(n_{1}\right) \cdots$ is not in $E$.

The next theorem is the first step towards our approximation theorem.

THEOREM 7.7. Let $E \subseteq \omega^{\omega} \times \omega^{\omega}$ be a set in $\mathscr{R}^{\rho}(\rho>0)$. Then there is a set $B \in \mathscr{B}_{0}^{\rho} \otimes \mathscr{B}_{\omega^{\omega}}$ such that $B \subseteq E$ and $B^{x}$ is comeager on $E^{*}$.

Proof. Let $R_{\rho}=R \Phi_{N}$ and assume $E=R \Phi_{N}\left(\left\{E_{n}\right\}\right)$, with each $E_{n}$ clopen. As in 6.3 , there are functions $f$ and $g$ such that

(i) $x \in E^{*} \leftrightarrow\left(\exists \eta_{0} \in M\right)\left(\forall n_{0} \in \eta_{0}\right)\left(\exists \eta_{1} \in M\right)\left(\forall n_{1} \in \eta_{1}\right) \cdots$

$$
(\forall k)\left[E_{\left\langle f\left(n_{0}\right) \ldots f\left(n_{k}-1\right)\right\rangle}^{x} \text { is comeager in } \Sigma\left(g\left(n_{0}\right) \hat{\cdots} g\left(n_{k-1}\right)\right)\right]
$$


where $M$ is such that $\Phi_{M} \sim \Phi_{N}$. Further, by Theorem 5.1, there is a $\mathscr{B}_{0}^{\circ}$-measurable function $x \mapsto \tau_{x}$ such that whenever $x \in E^{*}, \tau_{x}$ is a winning strategy for the player $\exists$ in the game (i). Moreover, by Lemma 7.5 and Remark 7.3.1, we may assume that the function $x \rightarrow \tau_{x}$ is such that for any complete play $\eta_{0}, n_{0}, \eta_{1}, n_{1}, \ldots$ agreeing with $\tau_{x}$ the sequence $\beta=g\left(n_{0}\right) \hat{g}\left(n_{1}\right) \hat{g}\left(n_{2}\right) \hat{\cdots}$ is in $E^{x}$, and that the base $M$ has been chosen as in Lemma 7.1. Define for each $k \geqslant 1$,

$$
W_{k}(s, x) \leftrightarrow \operatorname{Seq}(s) \& \ln (s)=k \&(\forall i<\operatorname{lh}(s))\left[(s)_{i} \in \tau_{x}(s \uparrow i)\right]
$$

i.e., $W_{k}^{x}$ consists of the codes of the first $k$ possible moves of $\forall$ when the existential player plays according to the strategy $\tau_{x}$. Clearly, $W_{k} \in \mathscr{B} \mathscr{R}_{0}^{\rho}$. Define

$C(x, y) \leftrightarrow(\exists \alpha)(\forall k)\left[W_{k}(\bar{\alpha}(k), x) \& y \in \Sigma\left(g(\alpha(0))^{\hat{\prime}} \cdots \hat{g}(\alpha(k-1))\right)\right] \& x \in E^{*}$.

Plainly, $C$ is the result of operation $\mathscr{A}$ on sets in $\mathscr{B} \mathscr{R}_{0}^{\rho} \otimes \mathscr{B}_{\omega^{\omega}}$. We shall show that (a) $C \subseteq E$ and (b) $C^{x}$ is comeager on $E^{*}$.

If $(x, y) \in C$ then $x \in E^{*}$ and $\exists$ wins the game (i). Further, there is a sequence $\left\{n_{h}: k \in \omega\right\}$ such that $n_{0}, n_{1}, n_{2}, \ldots$ are the moves of $\forall$ when $\exists$ plays according to $\tau_{x}$ and $y=g\left(n_{0}\right) \hat{g}\left(n_{1}\right) \cdots$. Consequently, $y \in E^{x}$. To prove (b), fix an $x$ such that $E^{x}$ is comeager. Then

$$
C^{x}=\left\{y \mid(\exists \alpha)(\forall k)\left[W_{k}(\bar{\alpha}(k), x) \& y \in \Sigma(g(\alpha(0)) \hat{\cdots} g(\alpha(k-1)))\right]\right\} .
$$

Define

$$
A_{\left\langle n_{0} \ldots, n_{k-1}\right\rangle}=\left\{\begin{array}{l}
\Sigma\left(g\left(n_{0}\right) \hat{g}\left(n_{1}\right) \hat{g} \hat{g}\left(n_{k-1}\right)\right) \quad \text { if } W_{k}\left(\left\langle n_{0}, \ldots, n_{k-1}\right\rangle, x\right), \\
\varnothing \quad \text { otherwise. }
\end{array}\right.
$$

Then, $C^{x}=\mathscr{A}\left(\left\{A_{s}\right\}\right)$. Hence to show $C^{x}$ is comeager, it is enough to show (by Vaught's Formula) that

$$
\begin{aligned}
& \left(\forall s_{0}\right)\left(\exists k_{0}\right)\left(\exists t_{0}\right)\left(\forall s_{1}\right)\left(\exists k_{1}\right)\left(\exists t_{1}\right) \cdots \\
& (\forall i)\left[A_{\left\langle k_{0} \ldots k_{t-1}\right\rangle} \text { is comeager in } \Sigma\left(\hat{s_{0} \hat{t}_{0} \cdots \hat{s_{i-1}} t_{i-1}}\right)\right] .
\end{aligned}
$$

So let $\forall$ play $s_{0}$. By Lemma 7.1, pick $k_{0} \in \tau_{x}(\langle\rangle)$ such that $g\left(k_{0}\right)$ extends $s_{0}$. Then $\exists$ replies with $k_{0}$ and a sequence number $t_{0}$ such that $\hat{s_{0} t_{0}}=g\left(k_{0}\right)$. Next when $\forall$ plays $s_{1}, \exists$ plays $k_{1} \in \tau_{x}\left(\left\langle k_{0}\right\rangle\right)$ such that $g\left(k_{1}\right)$ extends $s_{1}$ and then plays $t_{1}$ such that $\hat{s_{1}} t_{1}=g\left(k_{1}\right)$, and so on. By adopting this strategy, $\exists$ ensures that for each $i$,

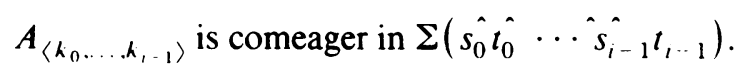

Thus, $C^{x}$ is comeager. An application of Lemma 7.2 gives the required set $B$.

The next lemma is the counterpart of Theorem 7.7.

Lemma 7.8. Let $E \subseteq \omega^{\omega} \times \omega^{\omega}$ be a set in $\mathscr{R}^{\rho}$. Let $E^{\#}=\left\{x: E^{x}\right.$ is meager $\}$. Then $E^{\#} \in c \mathscr{R}^{\rho}$ and there is a set $C \in \mathscr{B}_{R}^{\rho} \otimes \mathscr{B}_{\omega^{\omega}}$ (in fact, in $\sigma\left(\mathscr{R}^{\rho}\right) \otimes \mathscr{B}_{\omega^{\omega}}$ ) such that $E \subseteq C$ and $C^{x}$ is meager on $E^{\#}$.

Proof. Plainly, $E^{\#} \in c \mathscr{R}^{\rho}$, by Corollary 6.4. Let $f, g, N, M,\left\{E_{n}\right\}$ be as in Remark 7.6 with $\left\{E_{n}\right\}$ regular. Then, by (v) of Remark 7.6.

(i) $\quad E^{x}$ is meager $\leftrightarrow\left(\exists \eta_{0} \in M^{0}\right)\left(\forall n_{0} \in \eta_{0}\right)\left(\exists \eta_{1} \in M^{0}\right)\left(\forall n_{1} \in \eta_{1}\right) \cdots$

$$
(\exists k)\left[g\left(n_{0}\right) \hat{g}\left(n_{1}\right) \hat{\cdots} \notin E_{\left\langle f\left(n_{0}\right) \ldots \ldots f\left(n_{k}, 1\right)\right\rangle}^{x}\right] .
$$


Further, by Remark 5.1.1 there is a $\sigma\left(\mathscr{R}^{\rho}\right)$-measurable function $x \mapsto \tau_{x}$ such that $\tau_{x}$ is a winning strategy for $\exists$ in the above game whenever $x \in E^{\#}$. Moreover, $\tau_{x}$ can be chosen as in Remark 7.6. Call $u=\left\langle n_{0}, \ldots, n_{k-1}\right\rangle$ good with respect to $\tau_{x}$ and $\beta$ if $\beta$ extends $g\left(n_{0}\right) \hat{\cdots} \hat{g}\left(n_{k-1}\right)$ and $n_{0}, \ldots, n_{k-1}$ are the first $k$ possible moves of $\forall$ when $\exists$ plays with strategy $\tau_{x}$. Define

$$
\begin{aligned}
& T(u, x, \beta) \leftrightarrow \operatorname{Seq}(u) \&(\forall i<\operatorname{lh}(u))\left[(u)_{i} \in \tau_{x}(u \uparrow i)\right] \\
& \quad \& \beta \in \Sigma\left(g\left((u)_{0}\right) \hat{\jmath} g\left((u)_{1}\right) \hat{\cdots} \hat{g}\left((u)_{\operatorname{lh}(u)-1}\right)\right) \\
& \quad \&\left(\forall n \in \tau_{x}(u)\right)\left[\beta \notin \Sigma \left(g ( ( u ) _ { 0 } ) ^ { \wedge } g ( ( u ) _ { 1 } ) ^ { \wedge } g ( ( u ) _ { 2 } ) \hat { \cdots } \hat { g } \left((u)_{\operatorname{lh}(u)-1) \hat{g}(n))] .}\right.\right.\right.
\end{aligned}
$$

In other words, for each $x, \beta$, the section $T_{x, \beta}$ consists of all maximal good sequences with respect to $\tau_{x}$ and $\beta$. Clearly, $T \in \sigma\left(\mathscr{R}^{\rho}\right) \otimes \mathscr{B}_{\omega^{\omega}}$ and, moreover, $T^{u \cdot x}$ is closed nowhere-dense for each good sequence $u$ and $x$. Now define

$$
C^{\prime}(x, \beta) \leftrightarrow x \in E^{\#} \&(\exists u) T(u, x, \beta) .
$$

Set

$$
C=C^{\prime} \cup\left(\omega^{\omega} \mid E^{\#}\right) \times \omega^{\omega} .
$$

Since $E^{\#} \in c \mathscr{R}^{\rho}, C \in \sigma\left(\mathscr{R}^{\rho}\right) \otimes \mathscr{B}_{\omega^{\omega}}$; and to conclude the proof we shall show that $E \subseteq C$. So let $(x, \beta) \in E$. If $x \notin E^{\#}$, then we are done. So assume $x \in E^{\#}$. Therefore, $\exists$ wins the game (i) with strategy $\tau_{x}$. Now, suppose when $\exists$ plays the game (i) according to $\tau_{x}, \forall$ is able to play $n_{0}, n_{1}, n_{2}, \ldots$ such that for each $k,\left\langle n_{0}, \ldots, n_{k-1}\right\rangle$ is a good sequence with respect to $\tau_{x}$ and $\beta$. Then

$$
\beta=g\left(n_{0}\right) \hat{g}\left(n_{1}\right) \hat{\cdots}
$$

and hence, by Remark 7.6, $\beta \notin E^{x}$. Consequently, since we have $\beta \in E^{x}, \forall$ is able to play $n_{0}, n_{1}, \ldots$ 'consistent' with $\beta$ only up to a finite stage. Let $u$ be the code of the maximal sequence. Then $T(u, x, \beta)$ and hence $(x, \beta) \in C$.

The following gives the approximation at the first level.

Lemma 7.9. Let $E \subseteq \omega^{\omega} \times \omega^{\omega}$ be a set in $\mathscr{R}^{\rho}(\rho>0)$. Then there are sets $B, C$ in $\mathscr{B} \mathscr{R}_{0}^{\rho} \otimes \mathscr{B}_{\omega^{\omega}}$ such that $B \subseteq E \subseteq C$ and $C^{x}-B^{x}$ is meager for each $x$.

Proof. Let $T_{s}=E^{* \Sigma(s)} ; s \in$ Seq. As $E^{x}$ satisfies the Baire property for each $x$,

$$
\cup T_{s}=\left\{x: E^{x} \text { is nonmeager }\right\} \text {. }
$$

Since each $\Sigma(s)$ is a (recursive) homeomorphic copy of $\omega^{\omega}$, we can apply Theorem 7.7 to get $B_{s}$ as in the theorem. Put

$$
B=\cup B_{s} \cap\left(T_{s} \times \omega^{\omega}\right) .
$$

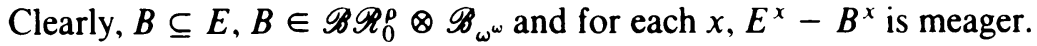

To get the set $C$, work with $E^{c}$ and argue as above using Lemma 7.8.

REMARK. Lemmas 7.7-7.9 are the analogues of Lemmas 1.3, 1.5 and 1.6 of Srivatsa for $C$-sets [17]. Naturally, from now on it is going to be a repetition of Srivatsa's techniques. 
Approximation Theorem 7.10. Let $A$ be an $\mathscr{R}_{\mu}^{\rho}$ subset of $\omega^{\omega} \times \omega^{\omega}(\rho>0$, $\left.\rho, \mu<\omega_{1}\right)$. Then there are sets $B$ and $C$ in $\mathscr{B}_{\mathscr{R}_{\mu}^{\rho}} \otimes \mathscr{B}_{\omega^{\omega}}$ such that $B \subseteq A \subseteq C$ and $C^{x}-B^{x}$ is meager for each $x$.

Moreover, if $A \in \mathscr{B} \mathscr{R}_{\mu}^{\rho}$, then one can find $B, C$ in $\mathscr{B}_{\mathscr{R}_{\mu}^{\rho}} \otimes \mathscr{B}_{\omega^{\omega}}$ with the above properties.

Proof. We shall prove this by induction on $\mu$. When $\mu=0$, this is just Lemma 7.9. So suppose $\mu>0$ and that the result is true for all $\nu<\mu$. Let $A \in \mathscr{R}_{\mu}^{\rho}$ and let $R_{\rho}=R \Phi_{N}$. Then $A$ is the result of operation $R_{\rho}$ on a family $\left\{A_{n}\right\}$ of sets from $c\left[\cup_{\nu<\mu} \mathscr{R}_{\nu}^{\rho}\right]$. By the induction hypothesis, for each $n$ we have $B_{n}$ and $C_{n}$ both in $\cup_{\nu<\mu} \mathscr{B} \mathscr{R}_{\nu}^{\rho} \otimes \mathscr{B}_{\omega^{\omega}}$ such that $B_{n} \subseteq A_{n} \subseteq C_{n}$ and $C_{n}^{x}-B_{n}^{x}$ is meager for each $x$. Let

$$
\tilde{B}=R_{\rho}\left(\left\{B_{n}\right\}\right) \text { and } \tilde{C}=R_{\rho}\left(\left\{C_{n}\right\}\right) \text {. }
$$

Then, $\tilde{B} \subseteq A \subseteq \tilde{C}$ and for each $x, \tilde{C}^{x}-\tilde{B}^{x} \subseteq \cup_{n}\left(C_{n}^{x}-B_{n}^{x}\right)$, and hence meager. To complete the proof it is enough to get $B \subseteq \tilde{B}$ and $C \supseteq \tilde{C}$ such that $B$ and $C$ are in $\mathscr{B}_{\mu}^{\rho} \otimes \mathscr{B}_{\omega^{\omega}}, \tilde{B}^{x}-B^{x}$ is meager and $C^{x}-\tilde{C}^{x}$ is meager for each $x$. We will show how to obtain $B ; C$ can be obtained similarly.

Let $\mathscr{F}$ be the $\sigma$-field generated by $\cup_{v<\mu} \mathscr{B} \mathscr{R}_{\nu}^{\rho}$. Then $\bar{B}=R_{\rho}\left(\left\{B_{n}\right\}\right)$ with each $B_{n}$ in $\mathscr{F} \otimes \mathscr{B}_{\omega^{\omega}}$. Thus, one can obtain a countably generated sub- $\sigma$-field $\mathscr{G}$ of $\mathscr{F}$ such that each $B_{n} \in \mathscr{G} \otimes \mathscr{B}_{\omega^{\omega}}$. Fix a countable generator of $\mathscr{G}$ and let $f:\left(\omega^{\omega}, \mathscr{G}\right) \rightarrow \omega^{\omega}$ be its characteristic function. Put $M=f\left(\omega^{\omega}\right)$. Then $\left(\omega^{\omega}, \mathscr{G}\right)$ and $\left(M, \mathscr{B}_{M}\right)$ are Borel isomorphic. For each $n$, let $B_{n}^{\prime}=\left\{(f(x), y):(x, y) \in B_{n}\right\}$. Then $B_{n}^{\prime} \in \mathscr{B}_{M \times \omega^{\omega}}$, for each $n$. Let $D=\{(f(x), y):(x, y) \in \tilde{B}\}$. Then $D=R_{\rho}\left(\left\{B_{n}^{\prime}\right\}\right)$. Hence, there is a set $E \subseteq \omega^{\omega} \times \omega^{\omega}$ in $\mathscr{R}^{\rho}$ such that $E \cap\left(M \times \omega^{\omega}\right)=D$. Apply 7.9 and get $\tilde{E} \subseteq E$ such that $\tilde{E} \in \mathscr{B}_{0}^{\rho} \otimes \mathscr{B}_{\omega^{\omega}}$ and $E^{x}-(\tilde{E})^{x}$ is meager for each $x$. Let $\tilde{f}: \omega^{\omega} \times \omega^{\omega} \rightarrow \omega^{\omega} \times$ $\omega^{\omega}$ be the map

$$
\tilde{f}(x, y)=(f(x), y) .
$$

Put $B=(\tilde{f})^{-1}(\tilde{E})$. Note that since $f$ is a bimeasurable map of $\left(\omega^{\omega}, \mathscr{G}\right)$ and $\left(M, \mathscr{B}_{M}\right)$,

$$
f^{-1}\left(\mathscr{R}^{\rho} \uparrow \omega^{\omega}\right) \subseteq\left\{A \subseteq \omega^{\omega}: A \text { is the result of operation } R_{\rho} \text { on sets in } \mathscr{G}\right\} \subseteq \mathscr{R}_{\mu}^{\rho} .
$$

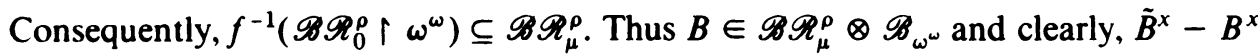
is meager for each $x$.

The second assertion follows from the first by observing that the class of all sets for which the result holds is closed under $\Phi_{N}$ and complementation.

The next proposition is quite well known and follows from the Von Neumann selection theorem.

Proposition. Let $(T, \mathscr{M})$ be a measurable space, $\mathscr{M}$ being a $\sigma$-field closed under operation $\mathscr{A}$, and let $Y$ be a Polish space. Let $B \in \mathscr{M} \otimes \mathscr{B}_{Y}$ have nonempty vertical sections. Then $B$ has an $\mathscr{M}$-measurable selection.

As a consequence of the approximation theorem we have the following

THEOREM 7.11. Suppose $A \subseteq \omega^{\omega} \times \omega^{\omega}$ is a $\mathscr{B} \mathscr{R}_{\mu}^{\rho}$ set $(\rho>0)$ such that $A^{x}$ is nonmeager for each $x$. Then $A$ has a $\mathscr{B} \mathscr{R}_{\mu}^{\rho}$-measurable selection. 
Proof. By Theorem 7.10, get $B \in \mathscr{B}_{\mu}^{\rho} \otimes \mathscr{B}_{\omega^{\omega}}$ such that $B \subseteq A$ and $A^{x}-B^{x}$ is meager for each $x . B^{x}$ is then nonempty for each $x$, and the above proposition yields the result.

The next selection theorem is due to Burgess [3].

THEOREM 7.12. Let $F: \omega^{\omega} \rightarrow \omega^{\omega}$ be a multifunction such that $F(x)$ is nonmeager in its closure $\operatorname{cl}(F(x))$. If $F$ is $\mathscr{B} \mathscr{R}^{\rho}$-measurable and its graph $\operatorname{Gr}(F) \in \mathscr{B}^{\circ}$, then $F$ has a $\mathscr{B} \mathscr{R}^{\rho}$-measurable selection.

Proof. Define $G$ by $G(x)=\operatorname{cl}(F(x))$. Then $G$ is a closed-valued, $\mathscr{B} \mathscr{R}^{\rho}$-measurable multifunction. Hence there is a map $g: \omega^{\omega} \times \omega^{\omega} \rightarrow \omega^{\omega}$ such that $g$ is $\mathscr{B} \mathscr{R}^{\rho} \otimes$ $\mathscr{B}_{\omega^{\omega}}$-measurable and $g(x, \cdot)$ is continuous, open and onto $G(x)$, for each $x$ (cf. [18]). Define $G^{\prime} \subseteq \omega^{\omega} \times \omega^{\omega}$ by

$$
G^{\prime}=\{(x, y): g(x, y) \in F(x)\} .
$$

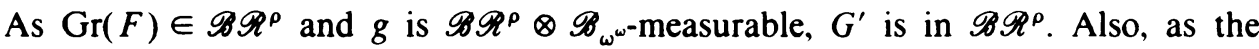
inverse image of a nonmeager set under a continuous open map is nonmeager, $G^{\prime}$ has nonmeager sections. By Theorem 7.11, $G^{\prime}$ has a $\mathscr{B} \mathscr{R}^{\rho}$-measurable selection $g^{\prime}$. Then $f(x)=g\left(x, g^{\prime}(x)\right)$ is a $\mathscr{B} \mathscr{R}^{p}$-measurable selection for $F$.

\section{REFERENCES}

1. P. Aczel, Quantifiers, games and inductive definitions, Proc. Third Scandinavian Logic Sympos. (S. Kanger, Ed.), North-Holland, Amsterdam, 1975.

2. J. P. Burgess, Classical hierarchies from a modern standpoint, Part I: C-sets, Fund. Math. 105 (1983), $81-95$.

3. 97-105.

4. , Classical hierarchies from a modern standpoint, Part II: R-sets, Fund. Math. 105 (1983), dam, 1982.

5. J. P. Burgess and D. E. Miller, Remarks on invariant descriptive set theon. Fund. Math. 90 (1975), $53-75$.

6. J. P. Burgess and R. Lockhart. Classical hierarchies from a modern standpoint, Part III: BP-sets, Fund. Math. 105 (1983), 107-118.

7. P. G. Hinman, Hierarchies of effective descriptive set theory, Trans. Amer. Math. Soc. 142 (1969), $111-140$.

8. Recursion-theoretic hierarchies, Springer-Verlag, Berlin and New York, 1978.

9. The finite levels of the hierarchy of effective R-sets, Fund. Math. 74 (1973), 1-10.

10. L. Kantorovitch and E. Livenson, Memoir on the analytical operations and projective sets. I, II, Fund. Math. 18 (1932), 214-279; 20 (1933), 54-97.

11. A. S. Kechris, Forcing in analysis, Higher Set Theory (Proc. Oberwolfach. Germany, 1977), Springer-Verlag, Berlin and New York, 1978.

12. A. A. Lyapunov, $R$-sets, Trudy Mat. Inst. Steklov. 40 (1953). (Russian)

13. On the classification of $R$-sets, Mat. Sb. 74 (1953), 255-262. (Russian)

14. Y. N. Moschovakis, Elementary induction on abstract structure, North-Holland, Amsterdam, 1974.

15. Descriptive set theory, North-Holland, Amsterdam, 1980.

16. B. V. Rao, Remarks on analytic sets, Fund. Math. 66 (1970), 237-239.

17. V. V. Srivatsa, Measure and category approximations for C-sets, Trans. Amer. Math. Soc. 278 (1983), 495-505.

18.

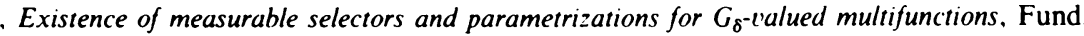
Math. (to appear).

19. R. L. Vaught, Invariant sets in topology and logic, Fund. Math. 82 (1974), 269-294.

Division of Mathematics and Theoretical Statistics, Indian Statistical Institute, 203, B. T. RoAD, CalCUTta 700035. INDIA 